\title{
Formation of Tropical Cyclones
}

\author{
By \\ Michio Yanai
}

Technical Paper No. 59

Department of Atmospheric Science

Colorado State University

Fort Collins, Colorado
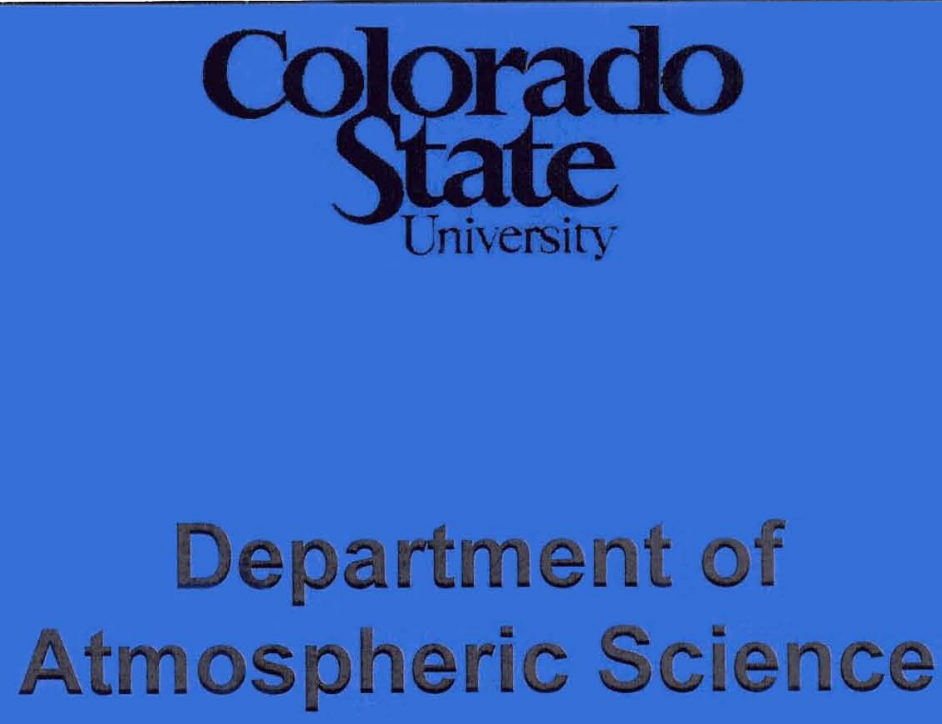

Paper No. 59 


\title{
Formation of Tropical Cyclones
}

\author{
Michio Y ANAi $^{1}$ \\ Department of Atmospheric Science \\ Colorado State University, Fort Collins
}

\begin{abstract}
What produces a hurricane or a typhoon? Despite years of effort by many meteorologists, the question has not yet been completely answered. To prepare a general background for understanding this problem, a brief survey is given of the structure and dynamics of fully developed tropical cyclones. Emphasis is given to the warm-core thermal structure, which has a significant role in driving the three-dimensional circulation, and to kinetic energy of mature tropical cyclones. Examination of the thermodynamic energy balance shows that the thermal structure is maintained by release of latent heat of condensation of water vapor. The importance of the processes taking place in the surface boundary layer is also discussed. Despite the apparent simple thermal structure of the mature cyclone, its formation process has turned out to be much more complex than it was once thought to be. A simple buoyant convective hypothesis will not adequately explain the observed fact; thus other factors become significant, especially large-scale flow patterns such as the equatorial shear zone, waves in the easterlies, and high-tropospheric migratory vortices. An analysis of the transient formative stage in which an originally cold-core wave disturbance is transformed into an incipient warm-core vortex is presented. According to this analysis, three characteristic stages in the whole process of cyclone formation are defined. Next we proceed to a summary of the progress of theoretical and numerical studies relating to tropical cyclone formation. The thermal (buoyant) instability theory, based on the negative vertical gradient of equivalent potential temperature, allows cumulus-scale convection but does not directly explain tropical cyclone formation. This has been confirmed by several numerical models using high-speed electronic computers. In reality, the tropical cyclone seems to possess two different convection characteristics, i.e., the macroscopic cyclone itself and cumulus clouds embedded within. Thus we need to advance a theory which explains the growth of the macroscopic cyclone by parameterizing the role of cumulus clouds. Two possible dynamical frameworks are considered. These are the 'balanced' and 'unbalanced' baroclinic vortex motions with a radial-vertical circulation. The latter theory involves the inertial instability mechanism along the vertically inclined isentropic surfaces. On the basis of facts and theoretical deductions, an attempt is made to develop a plausible model of tropical cyclone formation. The mechanisms considered important in each of the three postulated stages of formation are discussed. These are: (1) the pre-existing large-scale vertical motion associated with waves in the easterlies; (2) the organization of cumulus convection by the large-scale vertical motion and the role of the cumulus convection in heat liberation and vertical transport; and (3) the formation of a warm-core thermal field and the development of an incipient baroclinic vortex into a mature cyclone. Finally, the potential importance of several other studies, especially the study of mutual relationship between cumulus convection and the tropical cyclone, is mentioned. The need for expansion of observation networks in the tropics is emphasized in relation to several fundamental problems of tropical circulation.
\end{abstract}

1 On leave of absence from Meteorological Research Institute, Tokyo. 


\section{STRUCTURE AND DYNAMICS OF MATURE TROPICAL CYCLONES}

Tropical cyclones, i.e., hurricanes in the Atlantic, typhoons in the Pacific, and cyclones in the Indian Ocean, are the most violent large-scale convective systems in the atmosphere. To gain some insight into the formative mechanism of tropical cyclones, we begin our study with a discussion of the structure of a fully developed tropical cyclone and several fundamental hydrodynamical principles which govern the behavior of motion and energy conversion within the cyclone. This may provide us with some ideas about what should be studied in the formation problem.

Thermal and wind structure of mature tropical cyclones. One of the most interesting features of the tropical cyclone, other than its strong revolving mass, heavy precipitation, etc., is that the air above the central rainy region of the cyclone is much warmer than the surrounding air [Simpson, 1947; Palmén, 1948]. An idealized vertical cross section showing the temperature distribution in the tropical cyclone is reproduced from Palmén's paper (Figure 1). The scale along the ordinate is the logarithm of pressure, essentially equivalent with geometric height. Besides very warm air in the 'eye' of the hurricane, we note that relatively warm air also occupies a wide overlying region that has a radius of several hundred kilometers. The isotherms are inclined to the isobars which are horizontal in Figure 1. This characterizes the vortex as a 'baroclinic' disturbance. This will be explored later on.

Later studies on the temperature field in the cyclone have confirmed the validity of Figure 1 [Jordan and Jordan, 1954; Jordan, 1958b]. Observations made by specially instrumented research aircrafts of the National Hurricane Research Project of the United States Weather Bureau have further demonstrated this temperature distribution, especially in the central core region. Measurements in hurricane Daisy of 1958 [Colon et al., 1961] show a concentration of the lateral temperature gradient near the eye boundary which is even stronger than that

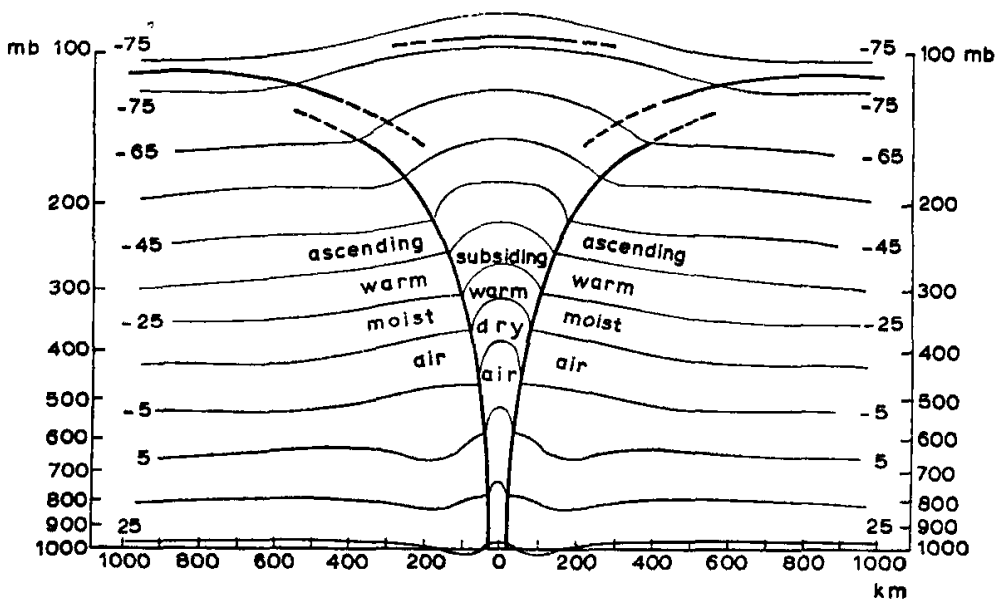

Fig. 1. Model of temperature (centigrade) distribution of mature tropical cyclone in vertical cross section [from Palmén, 1948]. 
indicated in Figure 1. Other evidence suggests that the eye wall is nearly vertical [Jordan et al., 1960].

The wind field in the mature tropical cyclone has also been studied [Hughes, 1952; Jordan, 1952]. Earlier results have been revised on the basis of recent aircraft observations. Figure 2 shows a typical example of the horizontal wind field observed within hurricane Donna of 1960 . Spirally converging inflow with counterclockwise rotation is seen in the lower level. On the other hand, clockwise rotating outflow is observed in the upper troposphere. In the central core region, the rotation is counterclockwise even at the upper level.

Role of the warm-core thermal structure in the dynamics of the tropical cyclone. We shall first present a set of equations to discuss certain dynamical principles and to relate the thermal structure and the wind field. In vector notation, the Eulerian equation of motion for a mass unit of air moving relative to the rotating earth is

$$
(d \nabla / d t)+2 \mathbf{\Omega} \times \nabla=-\nabla \Phi-\alpha \nabla p+\alpha \mathbf{F}
$$

where $\mathbf{v}$ is velocity, $\boldsymbol{\Omega}$ the rate of the earth's rotation, $\Phi$ the resultant potential of gravitation and centrifugal accelerations, $\alpha$ the specific volume, $p$ the pressure, and $\mathrm{F}$ the viscous force. The individual time derivative $d / d t=(\partial / \partial t)+\nabla \cdot \nabla$.

Conservation of mass is expressed by the equation of continuity

$$
\partial \rho / \partial t+\nabla \cdot(\rho \nabla)=0
$$

where $\rho(=1 / \alpha)$ is the density of the air.

The ideal gas law gives the equation of state for air,

$$
p \alpha=R T
$$

Here $R$ is the gas constant of air, and $T$ is temperature.
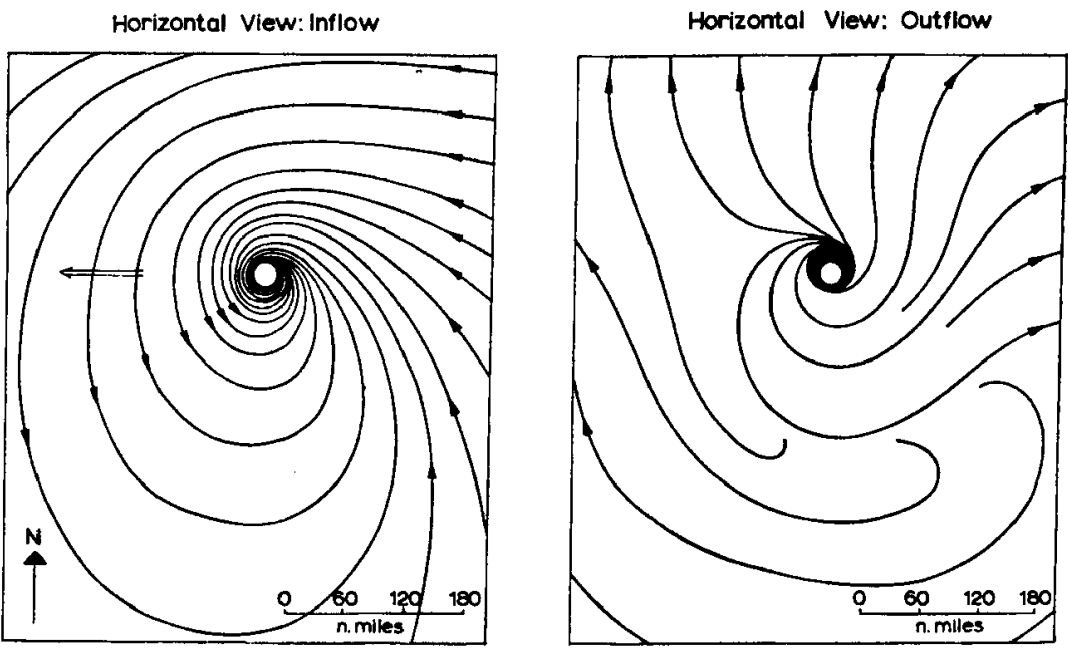

Fig. 2. Horizontal wind field of hurricane Donna of 1960. Left: lower troposphere. Right: upper troposphere. (Courtesy of H. Riehl.) 
For the ideal gas, the first law of thermodynamics is expressed by

$$
C, d T=d h-p d \alpha
$$

where $C_{v}$ is specific heat of air at constant volume. Equation 1.4 means that the increase of internal energy per unit mass of air $C_{\mathrm{b}} d T$ is due to the heat addition $d h$ and the work done by pressure $-p d \alpha$. By using (1.3), we find that (1.4) can also be expressed as

$$
C_{p} d T=d h+\alpha d p
$$

where $C_{p}$ is specific heat of air at constant pressure.

Equations 1.1-1.4 relate the four dependent variables (three components of $\nabla, p, \rho$, and $T$ ) with four independent variables, three in space and time, provided that the frictional force $\mathbf{F}$ is specified as a function of these variables.

In order to apply (1.1) to the dynamics of the tropical cyclone, it is convenient to use a cylindrical coordinate system locally fixed at the center of the cyclone which can be located at latitude $\phi$. Taking the radius $r$ positive outward, the azimuth $\lambda$ positive counterclockwise (origin at east), and the height $z$ positive upward, we can write the three component equations of (1.1) as

$$
\begin{aligned}
& \frac{d v_{r}}{d t}-f v_{\lambda}-\frac{v_{\lambda}{ }^{2}}{r}+\bar{f} w \cos \lambda=-\alpha \frac{\partial p}{\partial r}+\alpha F_{r} \\
& \frac{d v_{\lambda}}{d t}+f v_{r}+\frac{v_{r} v_{\lambda}}{r}-\bar{f} w \sin \lambda=-\alpha \frac{\partial p}{r \partial \lambda}+\alpha F_{\lambda} \\
& \frac{d w}{d t}-\bar{f}\left(v_{r} \cos \lambda-v_{\lambda} \sin \lambda\right)=-g-\alpha \frac{\partial p}{\partial z}+\alpha F_{z}
\end{aligned}
$$

where $v_{r}, v_{\lambda}$, and $w$ are the radial, tangential, and vertical velocities, respectively; $g$ is the acceleration of gravity; $F_{r}, F_{\lambda}$, and $F_{z}$ are the components of the viscous force; and $f=2 \Omega \sin \phi, \bar{f}=2 \Omega \cos \phi$ the vertical and lateral components of Coriolis parameter, respectively. Usually the terms involving $\bar{f}$ are neglected because they are 1 order of magnitude smaller than the other terms in each component equation.

As a first approximation, the air motion within the mature tropical cyclone can be regarded as a steady, frictionless, and axially symmetric vortex motion. This is given by

$$
\begin{aligned}
f v_{\lambda}+\left(v_{\lambda}{ }^{2} / r\right) & =\alpha(\partial p / \partial r) \\
-g & =\alpha(\partial p / \partial z)
\end{aligned}
$$

These are called the gradient wind and the hydrostatic equations, respectively. In the case of the gradient wind, the sum of Coriolis and centrifugal forces due to tangential motion balances the radial pressure gradient force. Differentiating and rearranging (1.7), we obtain the thermal wind equation

$$
\begin{aligned}
\left(f+\frac{2 v_{\mathrm{\lambda}}}{r}\right) \frac{\partial v_{\mathrm{\lambda}}}{\partial z} & =\left(\frac{\partial p}{\partial r} \frac{\partial \alpha}{\partial z}-\frac{\partial p}{\partial z} \frac{\partial \alpha}{\partial r}\right) \\
& =R\left(\frac{\partial \ln p}{\partial r} \frac{\partial T}{\partial z}-\frac{\partial \ln p}{\partial z} \frac{\partial T}{\partial r}\right)
\end{aligned}
$$


Equation 1.8 shows that the vertical variation of the tangential wind $v_{\lambda}$ is related to the solenoids formed by the intersections of isotherms and isobars in the $r-z$ plane (Figure 3). Because the solenoids are the result of warm-core thermal structure, we now know that the characteristic vertical wind variation in the tropical cyclone from counterclockwise below to clockwise above is related with the thermal structure of the cyclone.

The $\alpha-p$ solenoids in (1.8) are important for the dynamics of tropical cyclones. In order to discuss this, we shall generalize the measure of the solenoids and define the baroclinicity vector $B$ as

$$
\mathbf{B}=-\nabla \alpha \times \nabla p
$$

If the isobars and surfaces of equal specific volume are parallel (barotropic), $B$ vanishes. On the other hand, if these two surfaces are inclined relative to each other, the number of solenoids produced by the intersections will be given by the magnitude of $\mathbf{B}$. When we use the equation of state, $\mathbf{B}$ alternatively can be expressed as

$$
\mathbf{B}=\left(1 / \rho^{2}\right) \nabla \rho \times \nabla p=R \nabla \ln p \times \nabla T=\nabla \times(-\alpha \nabla p)
$$

Now we shall examine the role of the baroclinicity, i.e., the warm-core thermal structure, in its more general effects upon the air motion within the tropical cyclone. First we shall imagine a closed physical curve $L$ consisting of air particles. The curve can be chosen quite arbitrarily, as is illustrated in Figure 4, at a given instant in time. The chosen physical curve must preserve all the air particles henceforth. Therefore, except in the steady-state case, the shape of the curve will change with time.

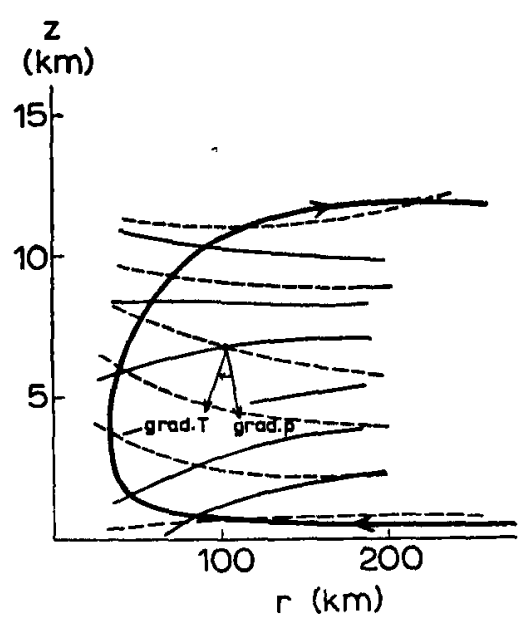

Fig. 3. Isobars (thin solid lines), isotherms (thin broken lines), and a stream line in radial-vertical plane of tropical cyclone.

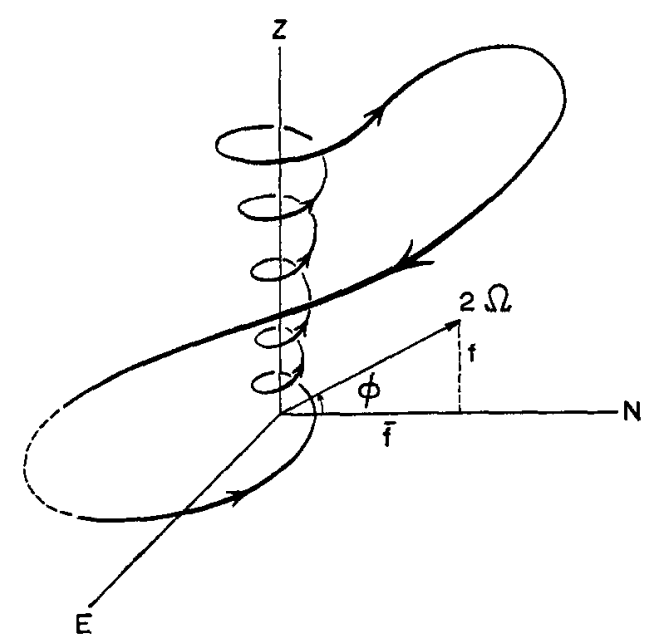

Fig. 4. An assumed physical curve consists of air particles in tropical cyclones. Vertical and lateral components of the earth's rotation vector 20 are also shown. 
Integration of the equation of motion (1.1) along the physical curve $L$ yields

$$
\oint_{L}\left(\frac{d \mathbf{v}}{d t}+2 \boldsymbol{\Omega} \times \mathbf{\nabla}\right) \cdot d \mathbf{r}=-\oint_{L} \alpha \nabla p \cdot d \mathbf{r}+\oint_{L} \alpha \mathbf{F} \cdot d \mathbf{r}
$$

As the line of integration is a material line, (1.11) can be transformed to

$$
\frac{d C}{d t}=-2 \mathbf{\Omega} \cdot \frac{d \mathbf{S}}{d t}+\int_{\mathbf{s}} \mathbf{B} \cdot d \mathbf{S}+\int_{\mathbf{z}} \nabla \times(\alpha \mathbf{F}) \cdot d \mathbf{S}
$$

where $C=\mathscr{S}_{L} \nabla \cdot d \mathrm{r}$ is the circulation along the curve, and $\mathrm{S}$ is the vector representation of the area enclosed by the curve $L$. Equation 1.12 is a general form of the circulation theorem of V. Bjerknes.

The first term on the right expresses an effect similar to magnetic induction. If a moving coil $(L)$ is in a magnetic field $(2 \Omega)$, the time change of the magnetic flux across the surface (S) will induce a time change of induction current $(C)$. Thus this effect entirely depends on the placing of the physical curve $L$ relative to the earth's rotation axis. The second term expresses the effect of baroclinicity. For an idealized axially symmetric model, the baroclinicity vector $B$ has only the component which appeared in (1.8). Without this component, i.e., $\alpha-p$ solenoids in the $r-z$ plane, the circulation cannot be intensified.

Maintenance of kinetic energy of tropical cyclone. The importance of the warm-core thermal structure will be better understood when we consider the maintenance of kinetic energy of tropical cyclones.

Taking the scalar product of (1.1) with $\nabla$, we obtain an expression for the time variation of mechanical energy,

$$
(d / d t)\left(\frac{1}{2} \mathbf{v}^{2}+\Phi\right)=-\alpha \nabla \cdot \nabla p+\alpha \nabla \cdot \mathbf{F}
$$

This equation states that the time rate of change of kinetic plus potential energy is not equal to zero, but that it is in fact equal to the work done by the pressure gradient force and the viscous dissipation. In a large-scale atmospheric system such as the tropical cyclone, the vertical component of velocity $w$ is, on the average, much smaller than the horizontal component $\nabla_{h}$. Therefore the kinetic energy per unit mass of air can be expressed as $k_{h}=\nabla_{h}{ }^{2} / 2$. The equation for the time rate of change of $k_{h}$ then can be obtained by taking the scalar product of (1.1) with $\nabla_{h}$,

$$
d k_{h} / d t=-\alpha \nabla_{h} \cdot \nabla p+\alpha \nabla_{h} \cdot \mathbf{F}
$$

2 Using the differential form of equation 1.12 by employing Stokes' theorem and defining the relative vorticity $\omega=\nabla \times v$, we can derive an equation for the time rate of change of the relative vorticity

$$
(\partial \omega / \partial t)+\nabla \times[(\omega+2 \Omega) \times \mathbf{v}]=\mathbf{B}+(\nabla \times \alpha \mathbf{F})
$$

or

$$
(\partial \boldsymbol{\omega} / \partial t)+(\boldsymbol{\nabla} \cdot \nabla) \mathbf{n}+\mathbf{n}(\nabla \cdot \mathbf{v})-(\mathbf{n} \cdot \nabla) \mathbf{v}=\mathbf{B}+(\nabla \times \alpha \mathbf{F})
$$

where $n=\omega+2 \Omega$ is the absolute vorticity. Equation $1.12 b$ shows that vorticity changes are produced by the advection of absolute vorticity, by the concentration of existing vorticity by divergence of air, by tilting of the vorticity vector in any nonuniform velocity field, by baroclinicity, and by the viscous effect. In meteorology the component of vorticity directed upward (perpendicular to the earth surface) is especially important. 
With use of the continuity equation 1.2,

$$
\left[\partial\left(\rho k_{h}\right) / \partial t\right]+\nabla \cdot\left(\rho k_{h} \nabla\right)=-\nabla_{h} \cdot \nabla p+\nabla_{h} \cdot \mathbf{F}
$$

When we apply (1.15) to the local cylindrical coordinate system, and assume the tropical cyclone to be a closed system, the time rate of change of total kinetic energy of the cyclone can be written

$$
\frac{\partial}{\partial t} \int_{V} \rho k_{h} d V=-\int_{V} \mathbf{v}_{h} \cdot \nabla p d V+\int_{V}\left(\nabla_{h} \cdot \mathbf{F}\right) d V
$$

where $d V=r d r d \lambda d z$ is the volume element. Equation 1.16 demonstrates that, for an increase of the total kinetic energy, the work done by the horizontal pressure gradient force must exceed the energy loss due to frictional dissipation. For the axially symmetric case,

$$
-\iint v_{r} \frac{\partial p}{\partial r} r d r d z \geqq-\iint\left(\mathbf{\nabla}_{h} \cdot \mathbf{F}\right) r d r d z
$$

is the requirement for maintenance of total kinetic energy.

Inequality 1.17 shows that $v_{r}$ and $\partial p / \partial r$ must have a negative correlation after integration over the entire domain. As is seen in Figure 3, this requirement is satisfied by the fact that the thickness between the upper and the lower isobaric surfaces increases toward the center. Since the thickness is proportional to the mean temperature of the air between the two surfaces by the hydrostatic relationship (equation 1.3 and the second equation of 1.7), we conclude that the storm must possess a warm-core structure for (1.17) to be valid. Thus a warm-core structure, i.e., a baroclinic field in the $r-z$ plane, is a necessary requirement for the maintenance of kinetic energy in the tropical cyclone.

Palmén and Jordan [1955] and Palmén and Riehl [1957] have made budget studies of total kinetic energy of a mean hurricane constructed from observations for various storms. The estimated production and dissipation of kinetic energy were almost identical, i.e., $150 \times 10^{18} \mathrm{ergs} \mathrm{sec}^{-1}$ when a tropospheric volume within a radius of $660 \mathrm{~km}$ was considered. The total storage of existing kinetic energy over the same domain was $12 \times 10^{24}$ ergs. Therefore the total kinetic energy of the storm can be created or dissipated when either of the two terms on the right-hand side of (1.16) acts for 24 hours at a constant rate and the other term vanishes.

Thermodynamic energy budget. Since an early study of Horiguti [1926, 1932], it has been accepted that the main energy source for the tropical cyclone is the release of the latent heat of condensation. However, it is only recently that meteorologists have been able to compute an approximate balance of total energy within the tropical cyclone based on observations.

The first law of thermodynamics can be generalized to include the mechanical and potential energy. The equation for the time rate of change of the total energy is obtained from (1.4) and (1.13),

$$
\begin{aligned}
\frac{d}{d t}\left(\frac{1}{2} \nabla^{2}+\Phi+C, T\right) & =-\alpha \nabla \cdot \nabla p-p \frac{d \alpha}{d t}+\frac{d h}{d t}+\alpha \nabla \cdot \mathbf{F} \\
& =-\alpha \nabla(p \nabla)+\frac{d h}{d t}+\alpha \nabla \cdot \mathbf{F}
\end{aligned}
$$


The time change in the sum of the kinetic, potential, and internal energy is due to the external work done by pressure force and the heat addition and the frictional dissipation. Using the continuity equation we can also write (1.18) in a flux form:

$$
(\partial / \partial t)\left[\rho\left(\frac{1}{2} \nabla^{2}+\Phi+C, T\right)\right]+\nabla\left[\left(\frac{1}{2} \nabla^{2}+\Phi+C_{p} T\right) \rho \nabla\right]=\rho(d h / d t)+\nabla \cdot \mathbf{F}
$$

In (1.19) the sum of flux of internal energy $C, T$ and the work done by pressure is expressed as the flux of enthalpy $C_{p} T$.

In a large-scale atmospheric motion, the kinetic energy is usually 2 orders of magnitude smaller than the other energy. Therefore it is practical to calculate the kinetic energy and the remaining energy separately. An approximate expression for the time rate of change of kinetic energy has already been obtained in (1.14). Then the remaining equation is

$$
(\partial / \partial t)[\rho(\Phi+C, T)]+\nabla\left[\left(\Phi+C_{p} T\right) \rho \nabla\right]=\rho(d h / d t)
$$

When heat sources other than the release of latent heat of condensation are neglected, (1.20) becomes

$$
(\partial / \partial t)[\rho(\Phi+C, T+L q)]+\nabla\left[\left(\Phi+C_{\nu} T+L q\right) \rho \nabla\right]=0
$$

where $L$ is the latent heat of condensation of a mass unit of water vapor. The specific humidity $q$ is the water vapor contained in a mass unit of air.

Considering a steady-state hurricane, the fluxes of potential energy, enthalpy, and latent heat must be balanced. Integrating (1.21) over a hurricane area with a radius of $200 \mathrm{~km}$, Palmén and Riehl [1957] obtained the values of energy flux shown in Table 1 . These values give us a picture of energy conversion occurring within the tropical cyclone. In the inner part of the cyclone, both latent and sensible heat are imported mainly in the lower layer, and they are converted to potential energy as the mass rises. The potential energy is exported to the surrounding atmosphere. The vital role of the latent heat in the energy budget is clear.

As we discussed before, the mechanical energy generation is $150 \times 10^{18} \mathrm{ergs} / \mathrm{sec}$ or $0.36 \times 10^{12}$ kilowatt-hours per day. Thus, if we regard the tropical cyclone as a heat engine driven by the release of latent heat of condensation, the efficiency of this engine, the ratio between the kinetic energy generation and the released heat, is about 3 per cent.

Heat and moisture transfer in the surface boundary layer. The tropical cyclone interacts strongly with the ocean surface. Therefore information on the energy

TABLE 1. Energy Flux for Mean Hurricane (1012 kilowatt-hours per day)*

\begin{tabular}{lrr}
\hline Inflow of latent heat & 13.3 & \\
Inflow of enthalpy & 22.8 & \\
$\quad$ Work done by pressure force & 7.7 & \\
Inflow of sensible heat & 15.1 & \\
Outflow of potential energy & & 36.4 \\
Balance & 36.1 & 36.4 \\
\hline
\end{tabular}

* Values taken from Palmén and Riehl [1957] 
exchange between air and ocean is required for an understanding of the maintenance of the cyclone.

It has been realized that the supply of heat from the ocean surface must be an important factor in explaining the observed surface temperature distribution in the tropical cyclone [Byers, 1944]. If we assume an adiabatic process along a trajectory of surface inflow, air temperature should be lowered toward the storm's center according to Poisson's equation

$$
T_{c}\left(p_{0} / p_{c}\right)^{(\gamma-1) / \gamma}=T\left(p_{0} / p\right)^{(\gamma-1) / \gamma}
$$

because pressure decreases toward the center. In (1.22), $\gamma=C_{p} / C_{v}, p_{c}$ and $T_{。}$ are central pressure and temperature, respectively, and $p_{0}$ is an arbitrary reference pressure. Observations have shown, however, that the sea level air temperature is almost constant within a storm.

Defining the potential temperature as

$$
\Theta=T\left(p_{0} / p\right)^{(\gamma-1) / \gamma}
$$

we can write the thermodynamic equation 1.4 as

$$
C_{\triangleright} d \ln \Theta=d h / T
$$

The right-hand term of (1.24) is the increase of entropy per unit mass of air. Thus the potential temperature is a measure of the entropy and a conservative property with respect to the adiabatic process.

For an isothermal condition realized in the surface air within the tropical cyclone, we must conclude that an addition of heat

$$
\begin{aligned}
\delta h & =C_{p} T(\delta \ln \Theta)_{T-\text { oonst }} \\
& =\alpha\left(p_{h}-p_{c}\right)
\end{aligned}
$$

is taking place. Here $p_{h}$ is pressure at the periphery of the cyclone.

Furthermore, to explain the observed very low surface pressure of the tropical cyclone, Malkus and Riehl [1960] have emphasized the importance of large amounts of moisture supplied from the ocean surface. In order to explain this, we must discuss the thermodynamics of moist air in which the condensation takes place.

Inserting the heat released by condensation into (1.24), we obtain the equation

$$
C_{p}(d \ln \Theta / d t)=-(L / T)\left(d q_{.} / d t\right)
$$

where $q$, is the saturation specific humidity. Because of the heat release, the potential temperature is no longer conservative in the saturated air.

We shall define a new conservative thermodynamic property in the saturated air as

$$
\Theta_{.}=\Theta \exp \left(L q_{\bullet} / C_{p} T\right)
$$

$\Theta$. is the equivalent potential temperature. Then (1.26) can be written

$$
d \ln \Theta . / d t=0
$$

Now we shall consider why the surface pressure in the tropical cyclone can achieve an extremely low value. It is not very rare to observe a storm whose 
central pressure is below $900 \mathrm{mb}$. From hydrostatic reasoning, it is obvious that this low surface pressure is the result of extreme warmness of the upper air. When the whole vertical column of air is composed of saturated air which has the same value of the equivalent potential temperature $\Theta_{00}$ of the surface layer, the maximum possible temperature increase may be reached.

Figure 5, taken from Malkus [1960], shows the temperature-pressure ascent curve in the central rain area of hurricane Daisy of 1958 . The curve is a composite of data from a radiosonde ascent and of measurements taken by research aircraft at several levels. For comparison, the distribution of the mean temperature in the West Indies taken from Jordan's [1958a] data is also shown in this figure. Significant warmer air is evident in the hurricane, especially in the upper and middle troposphere. In Figure 5 the dashed curve at the extreme right is the temperature distribution with constant equivalent potential temperature. This must correspond to the lowest obtainable surface pressure.

It has turned out, however, that, to explain the observed surface pressure in some fully developed cyclones, we must start with a very high equivalent potential temperature at the surface layer which is not observed in the usual undisturbed situation. Malkus and Riehl estimated that for a pressure decrease at sea level of $1 \mathrm{mb}$ we need an increase of $\Theta_{e}$ of $0.4^{\circ} \mathrm{K}$ if the vertical distribution of temperature within the hurricane is approximated by $\Theta_{0}=$ constant.

The mechanisms that make it possible to supply tremendous amounts of heat and moisture from the ocean surface to the air are considered to be the result of turbulent upward transport in the surface boundary layer. Terada [1955] and Fisher [1957] have estimated the sensible and latent heat exchange using Jacobs' [1942] semiempirical formulas

$$
\begin{aligned}
& Q_{o}=K_{\bullet}\left(T_{w}-T_{a}\right) V \\
& Q_{v}=K_{\varepsilon} L\left(q_{w}-q_{a}\right) V
\end{aligned}
$$

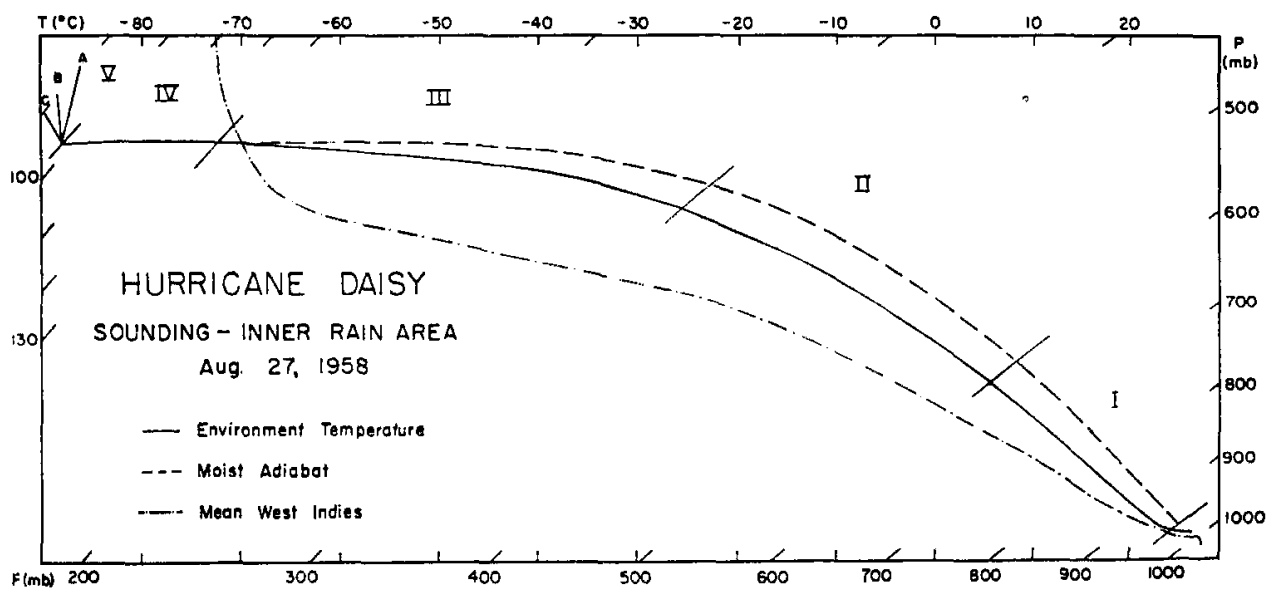

Fig. 5. Composite temperature-pressure sounding for inner rain area of hurricane Daisy of 1958 (solid curve). The dash-dotted curve shows the mean sounding in the West Indies area for hurricane season. The broken curve shows the temperatures of a moist adiabatically ascending air particle [after Malkus, 1960]. 
Defining the absolute angular momentum

$$
A=r\left(v_{\lambda}+\frac{1}{2} f r\right)
$$

we find that (1.34) becomes

$$
\begin{aligned}
& \frac{d v_{\tau}}{d t}=-\alpha \frac{\partial p}{\partial r}+A^{2} / r^{\mathrm{a}}-\frac{f^{2}}{4} r+\alpha \frac{\partial \tau_{r}}{\partial z} \\
& \frac{d A}{d t}=\alpha r \frac{\partial \tau_{\lambda}}{\partial z}=K \frac{\partial^{2} A}{\partial z^{2}}
\end{aligned}
$$

The second equation of (1.36) states that the absolute angular momentum will be conserved along an air trajectory if the frictional force vanishes. In the lower atmosphere within the tropical cyclone, however, the absolute angular momentum shows a remarkable decrease toward the center, indicating the importance of frictional effect.

For a steady-state nonviscous case, the tangential velocity distribution is obtained from $(d A / d t)=v_{r}(\partial A / \partial r)=0$ as

$$
v_{\lambda}=(C / r)-(f / 2) r
$$

where the integration constant $C$ will be determined by setting $v_{\mathrm{\lambda}}=0$ at an outermost radius $r_{m}$. In Figure 6, an ideal velocity distribution is shown together with model distributions constructed by Malkus and Riehl [1960] on the basis of observations. The differences between the theoretical wind distribution and the actual ones are systematic. For the ideal case, $v_{\lambda} r$ must tend to a constant with decreasing $r$. However, in reality $v_{\lambda} r^{x}=$ constant, where $x=0.6$ to 0.7 , is observed between $500 \mathrm{~km}$ and $200 \mathrm{~km}$. A sharp decrease of $v_{\lambda}$ is seen at the inner region.

The mechanical energy generation by the horizontal pressure gradient force in the boundary layer is compensated by the negative work done by the frictional force due to the shearing stress. The kinetic energy equation 1.15 can be written

$$
\left(\partial\left(\rho k_{h}\right) / \partial t\right)+\nabla \cdot\left(\rho k_{h} \nabla\right)=-\nabla_{h} \cdot \nabla p+\nabla_{h} \cdot\left(\partial \tau_{h} / \partial z\right)
$$

Integrating (1.38) over the boundary layer from the surface to the top $H$, where the stress vanishes, gives

$$
\begin{aligned}
\frac{\partial}{\partial t} \int_{0}^{B} \rho k_{h} d z+\int_{0}^{B} \nabla_{h} \cdot\left(\rho k_{h} \nabla\right) d z & +\left[\rho k_{h} w\right]_{0}^{B} \\
& =-\int_{0}^{B} \nabla_{h} \cdot \nabla p d z-\nabla_{h 0} \cdot \tau_{h 0}-\int_{0}^{B} \tau_{h} \cdot \frac{\partial \nabla_{h}}{\partial z} d z
\end{aligned}
$$

where $\tau_{h 0}$ is the stress at the surface. Thus the frictional dissipation can be divided into two parts, $-\nabla_{h 0} \cdot \tau_{h 0}$, the dissipation due to the surface stress, and $-\int_{0}{ }^{B} \tau_{h} \cdot\left(\partial \nabla_{h} / \partial z\right) d z$, the internal friction. The estimation of the internal friction is difficult and not yet established. The surface stress is empirically given by the formula

$$
\tau_{h 0}=k \rho_{0}\left|\nabla_{h 0}\right| \nabla_{h 0}
$$

where $k$ is a coefficient, and $\nabla_{h 0}$ is the wind at the surface. 
Here $Q_{\text {, and }} Q_{\text {o }}$ are sensible and latent heat fluxes in $\mathrm{cal} / \mathrm{sec} / \mathrm{cm}^{2}, K_{\text {, and }} K_{\text {. are }}$ the empirical exchange coefficients, $T_{w}-T_{a}$ the difference in degrees $\mathrm{C}$ between sea surface and ship deck level temperature, $q_{w}-q_{a}$ the difference in $\mathbf{g} / \mathbf{g}$ between the saturation specific humidity at the temperature of the sea surface and the actual specific humidity at ship deck level, and $V$ the wind velocity.

Malkus and Riehl [1960] have independently calculated the energy exchange from these formulas on the basis of a budget computation. The estimated heat pickup and latent heat pickup are $720 \mathrm{cal} / \mathrm{cm}^{2} /$ day and $2420 \mathrm{cal} / \mathrm{cm}^{2} /$ day, respectively, in the core region of a mature tropical cyclone. When we apply the results to formulas 1.29 and 1.30 , owing to strong wind velocity, these large amounts of energy exchange are possible even when we assume the usual exchange coefficients.

Momentum transfer in the surface boundary layer. Besides exchanging heat and moisture, the surface boundary layer also acts to exchange the momentum of air with that of oceanic water. Usually the air motion over the ground or ocean surface is not steady, laminar flow. Especially in the tropical cyclone, we observe gusty, turbulent wind which creates stresses in the boundary layer.

The horizontal stress acting on a unit volume of air is expressed as the vertical transport of momentum

$$
\tau_{h}=\rho \overline{w \nabla_{h}}=\rho \overline{w \nabla_{h}}+\rho \overline{w^{\prime} \nabla_{h}^{\prime}} .
$$

where the overbar means the average over a finite interval of time or space, and the prime denotes the deviation from the average. Because we can assume $\bar{w}=0$ in the boundary layer, the stress is given by

$$
\tau_{h}=\rho \overline{w^{\prime} \nabla_{h}^{\prime}}
$$

It is usually assumed that the horizontal stress $\tau_{h}$ is proportional to the vertical gradient of the mean horizontal motion, i.e.,

$$
\tau_{h}=\bar{\rho} K\left(\partial \bar{\nabla}_{h} / \partial z\right)
$$

where $\bar{\rho}$ is the mean density of air in the boundary layer, and $K$ is the coefficient of eddy viscosity. Equation 1.33 is no more than an approximation based on Prandtl's mixing length concept.

The role of the shearing stress for the air motion may be twofold. First, it acts as friction and causes dissipation of kinetic energy of the mean motion. Second, the stress induces the flow across the isobars. Thus the existence of the stress leads to the organization of a systematic mass transport and hence the vertical motion.

For simplicity, we shall consider the axially symmetric vortex motion and discuss the effects of shearing stress on the air motion within the vortex. Inserting the relation $\mathbf{F}_{\Lambda}=\partial \tau_{h} / \partial z$ into the horizontal components of (1.6),

$$
\begin{aligned}
& \frac{d v_{r}}{d t}-f v_{\lambda}-\frac{v_{\lambda}^{2}}{r}=-\alpha \frac{\partial p}{\partial r}+\alpha \frac{\partial \tau_{r}}{\partial z} \\
& \frac{d v_{\lambda}}{d t}+f v_{r}+\frac{v_{r} v_{\lambda}}{r}=\alpha \frac{\partial \tau_{\lambda}}{d z}
\end{aligned}
$$




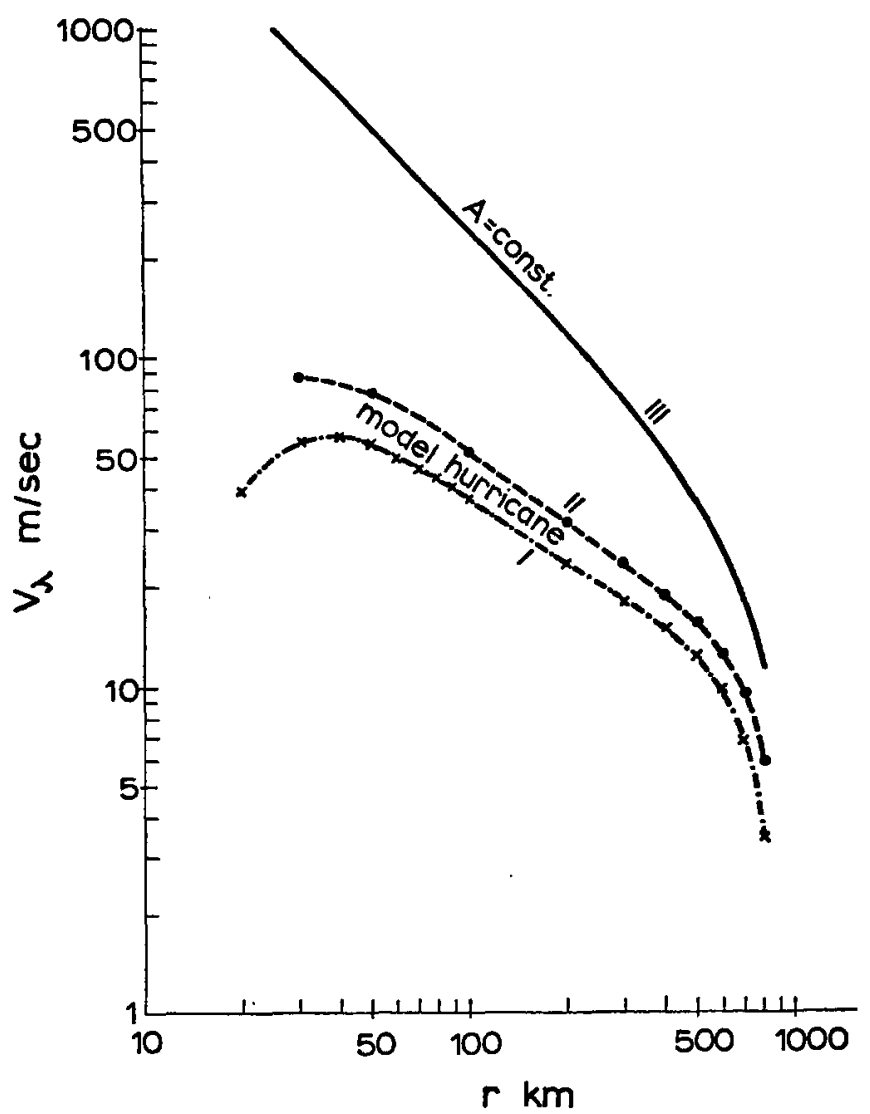

Fig. 6. Tangential wind speed (meters per second) in tropical cyclone. Curves I and II are based on the model proposed by Malkus and Riehl [1960]; curve III is for an ideal (nonviscous) vortex motion in which absolute angular momentum $A$ is constant ( $v_{\lambda}=0$ at $r=1000 \mathrm{~km}$ is assumed).

Mass transport due to the shearing stress. As was noted earlier, the steady-state vortex motion is approximated by the gradient wind equation $f v_{\lambda}+\left(v_{\lambda}{ }^{2} / r\right)=$ $\alpha \partial p / \partial r$. In the presence of friction, flow across the isobars will be induced. This effect must be large at the surface and may decrease with height. At some level in the free atmosphere the gradient wind relation may approximately hold. The vertical variation of the velocity within the friction layer was first discussed by Ekman [1905] for a wind-driven ocean current. Many variations of his derivation have been developed for the atmosphere. Assuming circular isobars, we shall follow Syōno's [1949] approximative treatment.

Defining $\zeta=\left(\partial v_{\lambda} / \partial r\right)+\left(v_{\lambda} / r\right)$ (the vertical component of the relative vorticity) and $P=p+\rho k_{h}$, we can write (1.34) as

$$
\begin{aligned}
-(f+\zeta) v_{\lambda} & =-\alpha(\partial P / \partial r)+K\left(\partial^{2} v_{r} / \partial z^{2}\right) \\
(f+\zeta) v_{r} & =K\left(\partial^{2} v_{\lambda} / \partial z^{2}\right)
\end{aligned}
$$


for the steady case. We shall replace $\zeta$ and $P$ by some suitable mean values over the boundary layer and insert the gradient wind equation for $P$. Then

$$
\begin{aligned}
-(f+\bar{\zeta}) v_{\lambda} & =-(f+\bar{\zeta}) v_{\sigma r}+K\left(\partial^{2} v_{r} / \partial z^{2}\right) \\
(f+\bar{\zeta}) v_{r} & =K\left(\partial^{2} v_{\lambda} / \partial z^{2}\right)
\end{aligned}
$$

where $v_{o r}$ is the gradient wind velocity.

We impose the boundary conditions such that the velocity tends to the gradient wind as we approach $z \rightarrow \infty$, and $\nabla_{h}=\kappa\left(\partial \nabla_{h} / \partial z\right)$ at the surface. The solution is given by

$$
\begin{aligned}
& v_{r}=-v_{\sigma r} \cdot C \exp \left(-\frac{\pi}{D} z\right) \cos \left(\frac{\pi z}{D}-\gamma\right) \\
& v_{\lambda}=v_{\theta r}\left[1+C \exp \left(-\frac{\pi}{D} z\right) \sin \left(\frac{\pi z}{D}-\gamma\right)\right]
\end{aligned}
$$

where

$$
\begin{gathered}
C^{2}=\left(1+2 \kappa \sqrt{(f+\bar{\zeta}) / 2 K}+\kappa^{2}(f+\bar{\zeta}) / K\right)^{-1} \\
\tan \gamma=1+(1 / \kappa) \sqrt{2 K /(f+\bar{\zeta})} \\
D=\pi \sqrt{2 K /(f+\bar{\zeta})}
\end{gathered}
$$

Equation 1.43 shows that the radial component is induced in the boundary layer and that the tangential component also deviates from the gradient wind. At the height $D$, the velocity becomes practically equal to the gradient wind velocity.

In vector form, (1.42) is

$$
-(f+\bar{\zeta}) \mathbf{k} \times\left(\mathbf{v}_{h}-\mathbf{v}_{g r}\right)=K \frac{\partial^{2} \mathbf{v}_{h}}{\partial z^{2}}=\alpha \frac{\partial \tau_{h}}{\partial z}
$$

where $\boldsymbol{x}$ is the unit vector pointing upward. Integrating this equation with respect to height up to $H$, where the stress vanishes,

$$
\int_{0}^{B} \tilde{\rho}\left(\nabla_{h}-\nabla_{o r}\right) d z=\frac{1}{f+\bar{\zeta}} \kappa \times \tau_{h 0}
$$

showing that the mass transport across the isobars is determined by the surface stress and the absolute vorticity $f+\bar{\zeta}$. Taking the horizontal divergence of the mass transport, we can estimate the frictionally induced vertical motion at the top of the boundary layer as

$$
\begin{aligned}
(\rho w)_{z-H} & =[1 /(f+\bar{\zeta})] \mathbf{k} \cdot \nabla \times \tau_{\tau_{00}} \\
& =[1 /(f+\bar{\zeta})](1 / r)\left(\gamma \tau_{\lambda 0} / \partial r\right)
\end{aligned}
$$

Thus the radial distribution of the stress determines the vertical velocity.

The frictionally induced vertical motion is not the only reason for the vertical motion in the tropical cyclone. Nevertheless, it undoubtedly contributes to the organization of strong vertical motion. This view has been emphasized by Syono et al. [1951] and Eliassen [1959]. 
In order to discuss the maintenance of the steady-state mature tropical cyclone, we need to relate the heat and moisture supply and the momentum loss in the boundary layer. We also have to relate the surface boundary layer to the upper free atmosphere. Very recently, Riehl [1963] has developed a theory relating the momentum loss and the heat and moisture supply from the ocean surface and explaining some features of the thermal and wind structure of the tropical cyclone.

It is generally considered that the formation of the 'eye' of the tropical cyclone is essentially the result of centrifugal force due to strong rotation. Syono [1951] has computed the diameter of the eye using the law of conservation of absolute angular momentum. Kuo [1959] has revised the result by including the loss of angular momentum within the boundary layer.

\section{OBSERVATIONAL STUDIES OF \\ TROPICAL CYCLONE FORMATION}

We now proceed to the main problem of cyclone formation. Although this problem must be approached from a variety of viewpoints, the introduction may have provided us with some basic understanding of the physical aspects of the problem. Let us consider the problem in the following manner. When and by what mechanisms is the large warm-core vortex created in the tropical atmosphere?

Origin of the tropical cyclone. Climatologically, it is known that the formation of a tropical cyclone is confined within particular geographical regions and seasons. Figure 7 shows the marked correlation between cyclone formation and sea surface temperature. Detailed statistics of cyclone formation in particular areas will be found in various publications, such as those of Dunn and Miller [1960], Ramage [1959], Arakawa [1963], Gabites [1963], Gentry [1963], and Koteswaram [1963].

Palmen [1948] has demonstrated the threshold temperature of the sea surface necessary for tropical cyclone formation. Tropical cyclones are formed only over the ocean where surface temperatures exceed about 26 to $27^{\circ} \mathrm{C}$. Undoubtedly the air over the warm and moist ocean surface has a high value of equivalent potential temperature $\Theta$. (see equation 1.27). When an air particle is lifted beyond the condensation level, release of latent heat will increase the potential temperature of the air in such a way that the equivalent potential temperature is conserved. If the resulting air particle temperature is higher than that of the surrounding

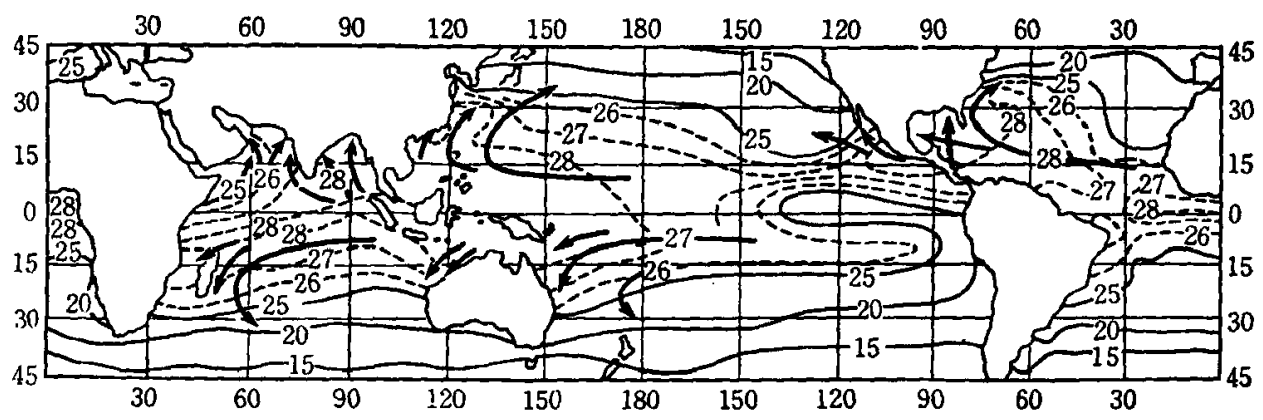

Fig. 7. Principal tropical cyclone paths and sea surface temperature during summer season (for both hemispheres) [after Palmén, 1948]. 
air, the parcel may be accelerated upward further by the buoyancy force (Figure 8).

The third equation of motion can be written, using the excess of the temperature of the rising air parcel over the temperature of the environment, as

$$
d w / d t=g\left(T^{\prime}-T\right) / T
$$

where friction is neglected. Here $T^{\prime}$ is the temperature of a buoyant air particle, and $T$ is the surrounding temperature. When we integrate the temperature excess over the path of the particle from sea level to the top of the atmosphere, the result gives the total latent energy which can be converted into kinetic energy of the rising air particle. This is expressed by

$$
W=\frac{1}{2} w^{2}=g \int_{0}^{s} \frac{\left(T^{\prime}-T\right)}{T} d z=-R \int_{p_{0}}^{p}\left(T^{\prime}-T\right) \frac{d p}{p}
$$

Graphically, the integral $W$ is expressed by the area enclosed by the $p-T$ curve of the particle and that of the surrounding air (Figure 8).

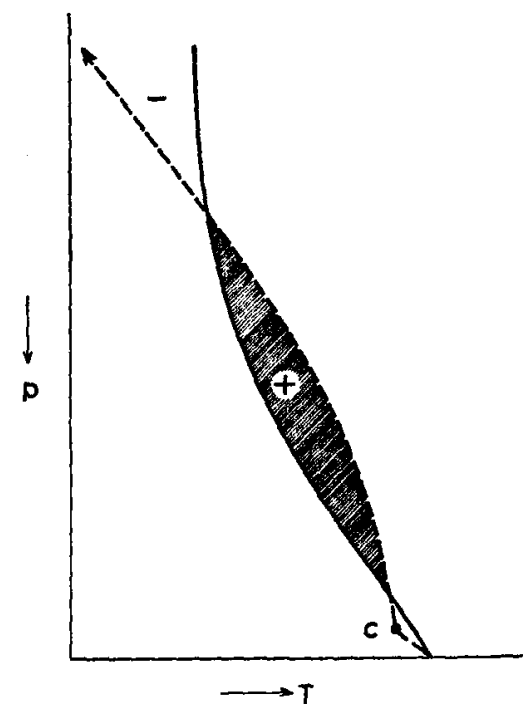

Fig. 8. Model of latent-unstable atmosphere. When the vertical gradient of air temperature is between those of dry and moist adiabatic lapse rates, a particle forced to lift from the sea surface first will meet cooling up to the condensation level $(C)$. Then the release of latent heat of condensation lowers the cooling rate. Eventually the particle temperature becomes warmer than the surrounding air temperature and the particle becomes buoyant.

Palmen [1948] investigated the latent energy distribution over the Atlantic and found that the distribution has a remarkable variation with season and location. This variation closely approximates the annual change of hurricane formation. A similar study was made by Kasahara [1954] in the Pacific.

This correlation does not necessarily mean that the formation of tropical cyclones is directly explained by a simple buoyant convective process. As we will see, the buoyancy concept is applicable to many scales of thermal convection. It has recently been established that the expected horizontal scale of the convection produced by thermal buoyancy does not explain the observed scale of the tropical cyclone.

Nevertheless, the climatological facts suggest that the availability of latent energy does have a fundamental role in tropical cyclone formation, although it may not be in the direct manner considered before but in an implicit manner. The recognition of cumulus convection as an integral part of tropical cyclones, 
and also the recognition of a difference between the mechanisms driving cumulus clouds and the tropical cyclone itself, has recently been confirmed by several observational and theoretical studies.

The equatorial shear zone. Present knowledge of disturbances in the tropical atmosphere are largely due to the expansion of the upper-air observation networks over the tropical Atlantic and the Pacific since about 1940.

Depperman [1936] already had noticed traveling disturbances in the western Pacific, where a pronounced equatorial shear zone exists. The shear zone separates the trade easterlies to the north and westerlies to the south.

A network covering the Caroline, Mariana, and Marshall Islands was developed by the Central Meteorological Observatory and Navy Hydrographic Office of Japan before World War II. In 1939 and 1940, an especially dense pilot balloon observation network of about 30 stations was organized for the study of typhoons. Using these data, Uwai [1943] and Otani [1953] have made extensive studies of the socalled equatorial front (shear zone) and its relation to typhoon formation.

The term 'front' was used in analogy to the polar front. Present views consider this terminology incorrect, however, because there is no marked horizontal temperature gradient in the shear zone. This zone is characterized by a remarkable horizontal wind shear, which sometimes yields a sharp discontinuity in the wind field observed on the weather map. Most typhoons originate in the vicinity of this transition zone, especially from wave-type disturbances traveling along this zone. Another important aspect of the equatorial shear zone is its abundance of moisture. In Figure 9 the wind system in the vicinity of the equatorial shear zone is illustrated.

In the Atlantic, too, we have a transition zone separating the easterlies along the southern periphery of the Azores-Bermuda subtropical high from the southeasterlies coming from the southern hemisphere. However, the lack of suitable observation stations has hampered extensive research on the shear zone in the Atlantic.

Waves in the easterlies. Despite the difficulty of observation in the vicinity of the equatorial shear zone, the modern study of wave disturbances has advanced first in the Atlantic Caribbean region. The existence of disturbances in the tropical Atlantic traveling westward from the African coast was mentioned by Dunn [1940].

Riehl [1945, 1948a] has made extensive studies of these disturbances in the Caribbean region and has developed the concept of the easterly wave. Easterly waves are found in the lower troposphere of the tropics, and they travel along the trade wind. Most hurricanes develop from these waves. In Figure 10 a schematic horizontal view of the stream lines associated with an easterly wave is shown. Generally speaking, easterly waves are associated with shower activity which occurs east of the wave axis. The axis (crest) of the wavy stream-line pattern usually shifts toward the east with increasing height. The rear part of the wave possesses colder air aloft than its surroundings. Yet this colder air is located in the place of upward motion and precipitation. Riehl concluded that the waves are essentially cold-core disturbances. This is quite a paradoxical finding because we know that mature tropical cyclones are warm-cored. This finding presents one of the major difficulties in explaining tropical cyclone formation by the buoyant convective hypothesis.

The study of easterly waves has been extended for the Pacific by Riehl [1948b] 


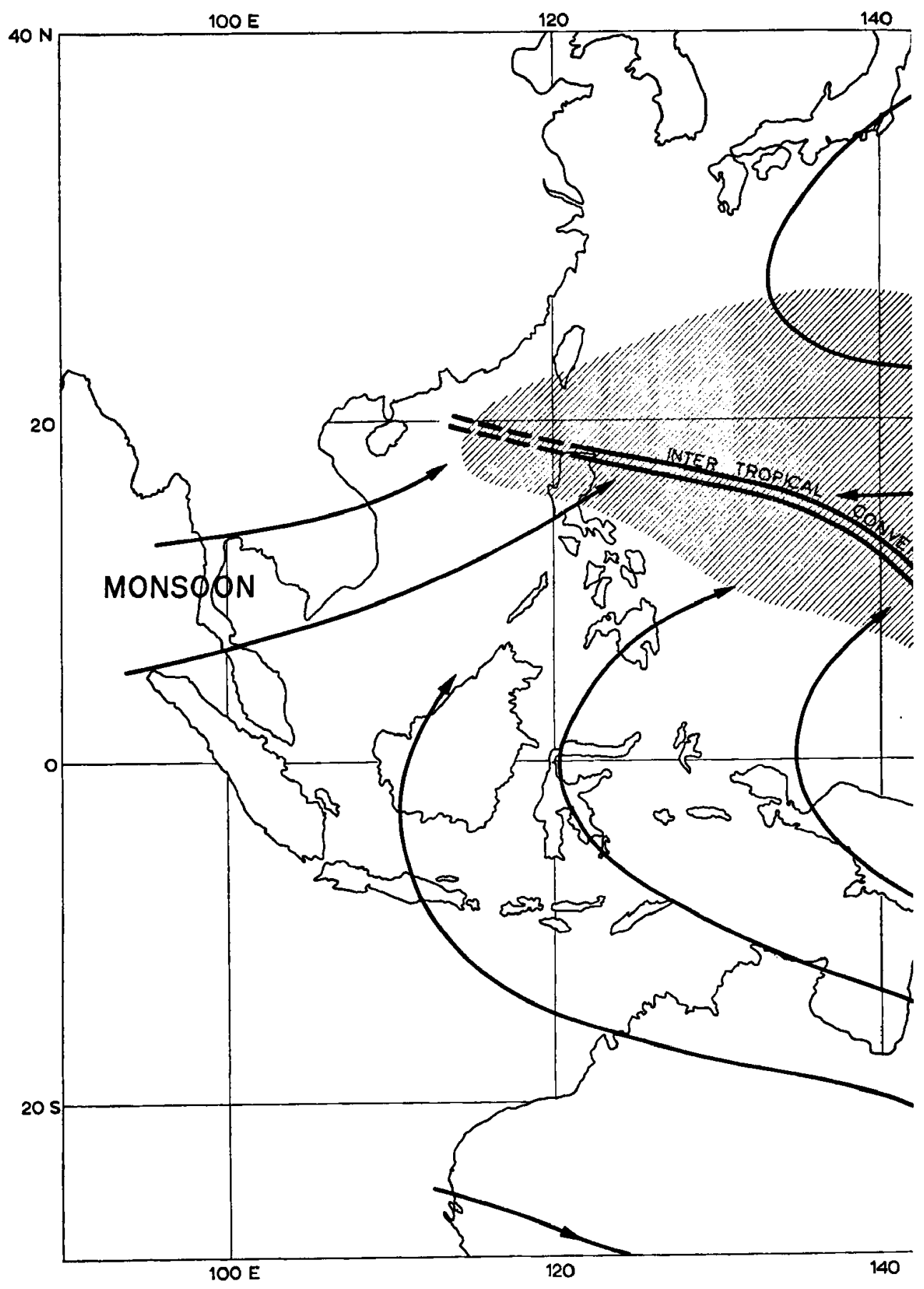

Fig. 9. Schematic view of the equatorial shear zone in the western Pacific 


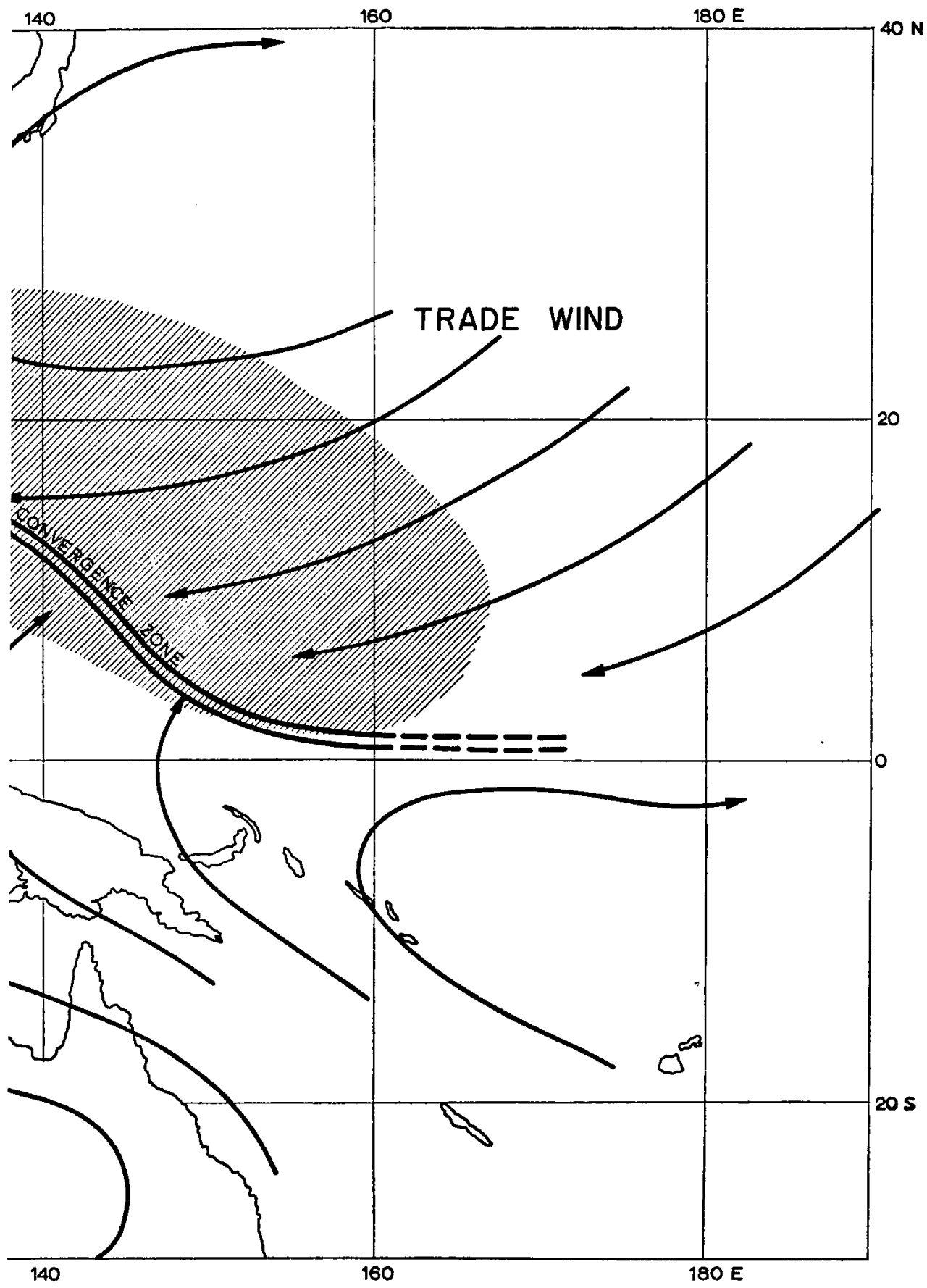

in northern summer. Typhoon generating region is shown by shaded areas. 


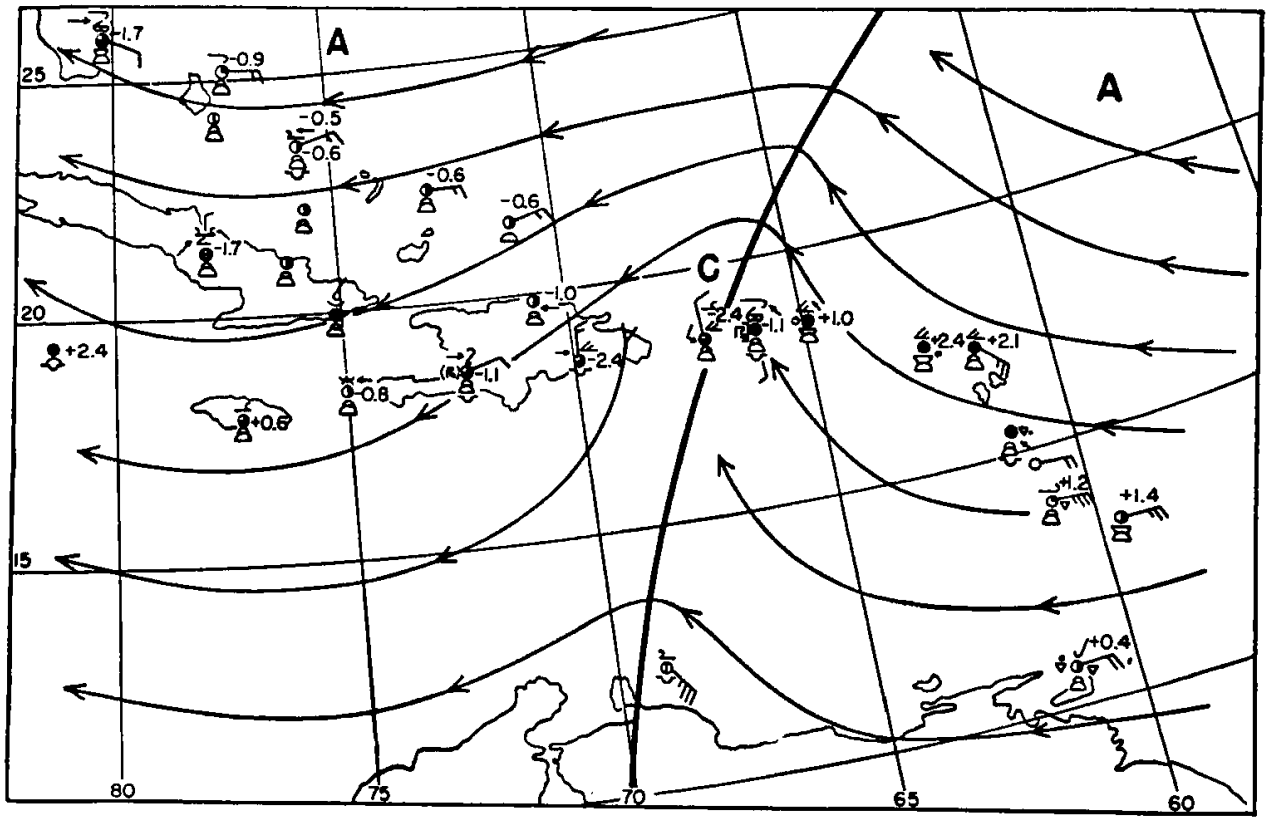

Fig. 10. Example of stream lines associated with an easterly wave over the Caribbean area [after Riehl, 1948a].

and Palmer [1952]. The above-mentioned characteristics of the easterly wave are now generally accepted. It should be mentioned that not all waves in the easterlies transform themselves into tropical cyclones. The frequency of easterly waves is quite high. Roughly speaking, we can expect a wave passage over a tropical island station once every three or four days. However, tropical cyclones are comparatively rare phenomena.

Many studies have been made of the factors that might control the development of the easterly wave into the tropical cyclone. Riehl [1948b] found a close correlation between the upper tropospheric flow patterns and storm development. He has emphasized the importance of mass divergence in the upper troposphere favored by large-scale migratory clockwise rotating vortices with strongest concentration near the 200-mb isobaric surface. McRae [1956] and Koteswaram and George [1957] have reported similar synoptic features for formation of South Pacific typhoons and cyclones in the Bay of Bengal. I [Yanai, 1964] have also investigated relationships between the upper tropospheric flow pattern and the development of easterly waves in the equatorial Pacific. Figure 11 shows the passages of upperlevel anticyclones and low-level wave disturbances over the Marshall Islands area. It can be seen that the waves which later developed into typhoons were located under the high-level anticyclones. From examination of seventeen easterly waves during June and July of 1958 in the Marshall Islands area, we found that intensification took place in six cases, all of them showing the same sequence of evolution. However, a reverse statement is not valid. The existence of an upperlevel anticyclone is necessary but not sufficient for the development. 

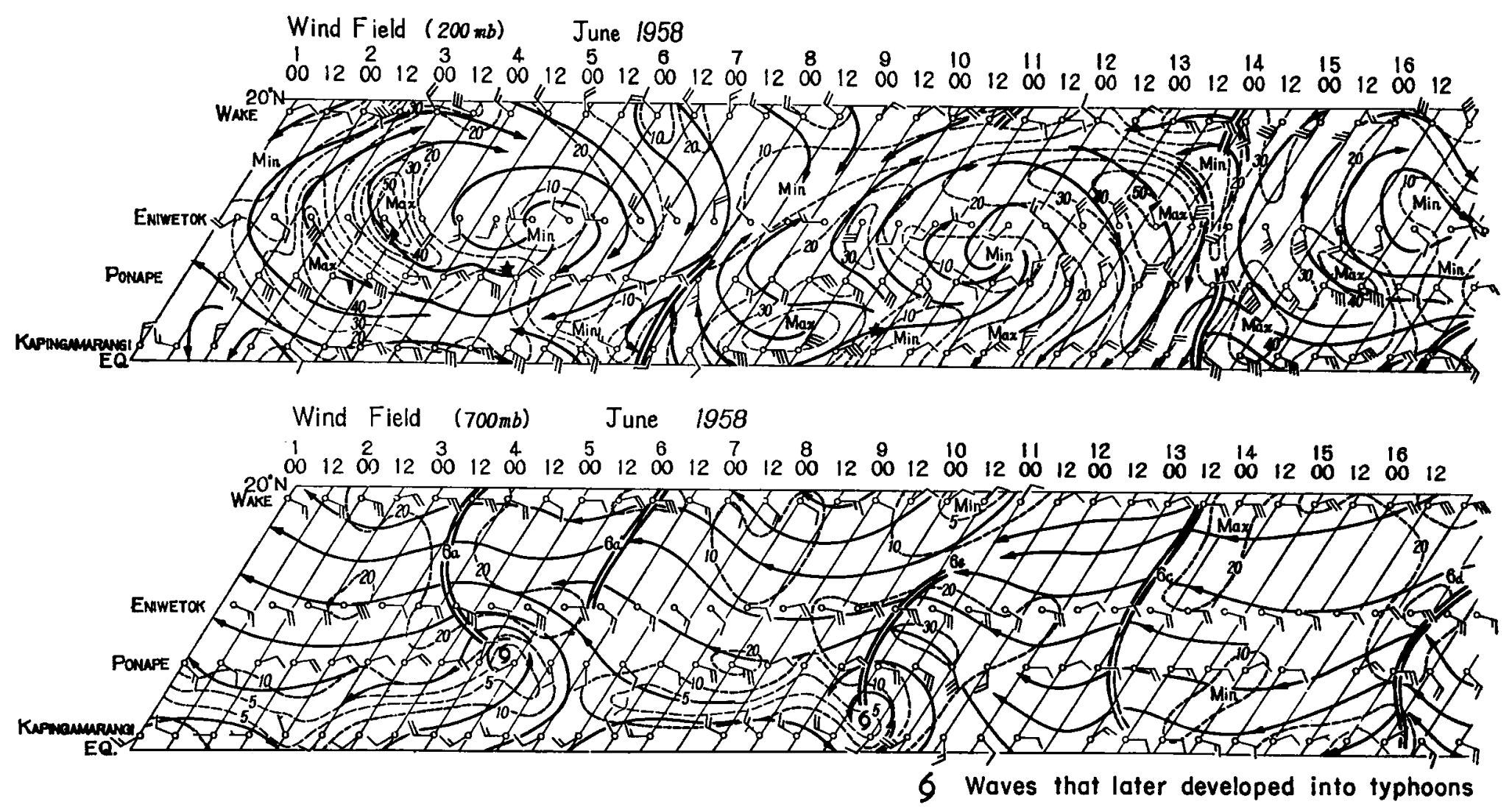

Fig. 11. Horizontal time sections showing passages of migratory vortices at $200 \mathrm{mb}$ (top) and those of waves at $700 \mathrm{mb}$ (bottom) over the Marahall Islands stations, June 1958. 
On the other hand, Riehl [1948a] has mentioned that tropical cyclone formation is also affected by middle-latitude disturbances. He noticed several times that waves in the easterlies and waves in the middle-latitude westerlies mutually interact and that both disturbances intensify. According to Riehl et al. [1964], existence of an extended trough from middle latitudes is one of the important factors favorable to formation of hurricanes in the Gulf of Mexico.

Transformation process. There is a need for more precise observations on the transient stage of development from a wave disturbance to an incipient tropical cyclone. Although several studies have been presented [Hubert, 1955; Riehl and Gentry, 1958], the main obstacle for this sort of study continues to be poor distribution of upper-air stations over wide oceanic areas.

' Within my knowledge, the most densely distributed observation network at the present is that of the Marshall Islands area operated during the atomic and nuclear tests by the U. S. Joint Task Force. Using data from this network, Palmer [1952] was able to present his extensive survey of equatorial easterly waves. He described some of the dynamical features of the waves, such as the distribution of the horizontal divergence, and also gave a qualitative description of the transformation process from wave into typhoon.

I was fortunate in obtaining observations of the formative stage of typhoon Doris over the network of the Marshall Islands in July 1958. The analysis was mainly concerned with the period of transition from an easterly wave with coldcore thermal structure to its development as an incipient vortex of warm-core type. The following is an extract from my analysis [Yanai, 1961a, b; 1963]. Figure 12 is a schematic presentation of the transformation process.

(a) Wave Stage (Initial)

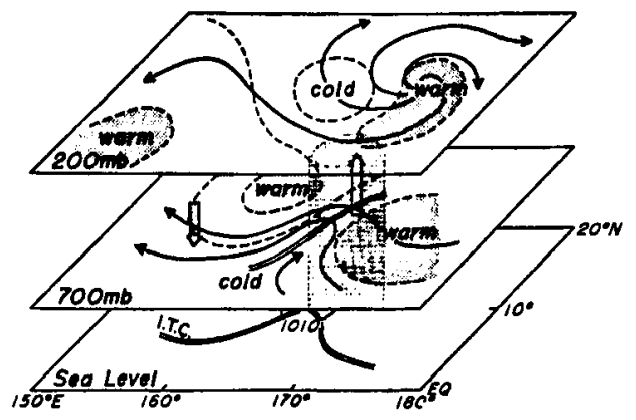

(b) Worming Stage (24hour later)

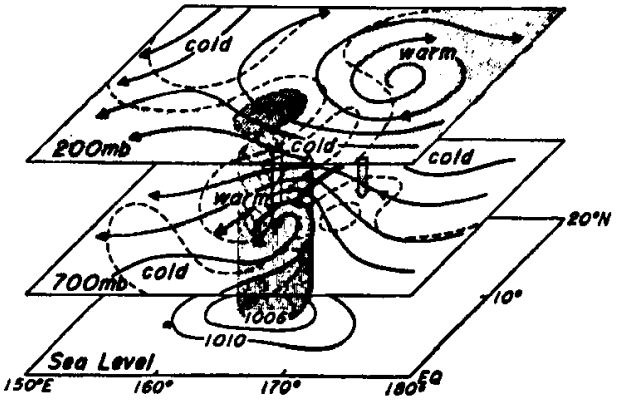

(c) Developing Stage (48hour later)

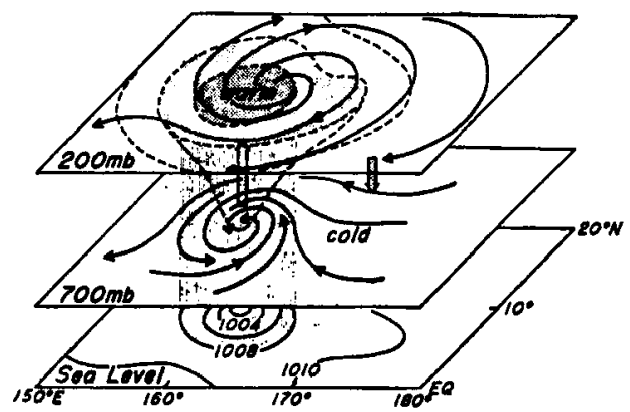

Fig. 12. Model of typhoon formation process constructed from observation for typhoon Doris of 1958: (a) the wave stage with cold ascending air; (b) the warming stage; (c) onset of the developing stage. Relatively (horizontally) warm area and precipitation are shown by shaded areas. 
Initially (Figure 12a) the easterly wave acquired a large amount of vorticity, and there existed a well-defined storm region. The center of the upward motion (as computed from the continuity equation) was slightly behind the axis of the wave, where cold air, especially in the upper troposphere, was also situated. Further eastward, an intense upper-level anticyclonic cell with outflow was present.

Twenty-four hours later, the low-level wave amplified and a weak vortex formed (Figure 12b). Simultaneously, warming of the upper air occurred. This was the result of release of latent heat of condensation, as was confirmed through a budget study of heat and moisture. The warm core appeared first in the layer between 300 and $400 \mathrm{mb}$, then gradually expanded downward. As a consequence of the warming, the upper anticyclone intensified and came to be located directly above the low-level disturbance.

Two days after the initial time, the warm-core structure had become completely established throughout the troposphere. Major characteristics of a typhoon, i.e., inflow with cyclonic rotation in the low levels and anticyclonic outflow in the upper levels, were now established. However, even at this stage, the central pressure at sea level had decreased only slightly (Figure 12c).

During the decisive change of thermal structure from cold to warm core, the order of magnitude of vertical velocity and of vorticity did not change abruptly. However, when the warm-core structure was completed, the central pressure decreased rapidly. Figure 13 shows the change of central pressure. Analysis of the equivalent potential temperature showed unstable stratification with respect to the moist adiabatic process at the beginning. This changed to almost neutral stability when the rapid development started.

I have defined three stages in the whole process of tropical cyclone formation. This was done for convenience of description and theoretical interpretation. The first stage is the intense easterly wave. The wave possesses vorticity and vertical motion not very different from those of an incipient tropical cyclone in magnitude and horizontal extension. The main problem of the wave stage may be explanation

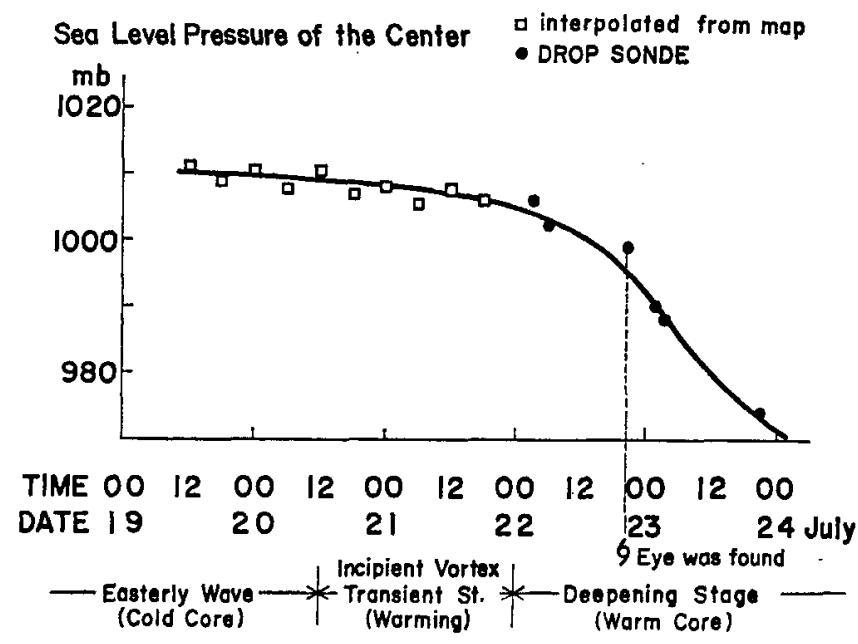

Fig. 13. Change of the central sea level pressure of typhoon Doris of 1958 during its formative stages. 
of the cold-core structure, especially with respect to maintenance of the kinetic energy.

In the second stage the warming of the upper troposphere occurs, resulting in the transformation of the cold core to a warm core. There is no doubt about the importance of condensation heating in this stage. However, it is difficult to apply the simple buoyancy concept directly to the observed large-scale vertical motion.

The third stage is the development into a mature tropical cyclone after the warm-core structure has been established. To prepare the reader for further discussion, we shall go into some fundamental theoretical considerations of the problem.

\section{THEORETICAL AND NUMERICAL STUDIES}

None of the existing theories covers all aspects of tropical cyclone formation. Most of them are concerned with stability criteria leading to a growing convection in the tropical atmosphere. There are two basic categories of mechanisms which have been considered important in tropical cyclone formation. These are the thermal buoyant instability and the inertial instability mechanisms.

Thermal (buoyant) instability theories. As was mentioned before, the thermal structure of a tropical cyclone is considered to be the result of release of latent heat of condensation. Several authors have attempted to develop a mathematical theory of tropical cyclone formation which includes the condensation process [Haque, 1952; Kasahara, 1952; Syōno, 1953].

In the real atmosphere, the main cause of thermal instability is not a direct negative vertical gradient of temperature, but a combined distribution of temperature and moisture. However, so far as the mathematical treatment is concerned, these theories are essentially equivalent to those of classical Rayleigh convection which is induced by heating from below.

We consider a layer of liquid whose depth is $d$. A constant negative vertical temperature gradient has been imposed upon this liquid layer (Figure 14). It is well known that, when the temperature gradient $(\partial T / \partial z<0)$ exceeds a certain value, convection begins and the so-called Bénard cells are formed. According to Rayleigh [1916], the onset of convection is determined by a nondimensional number $R_{a}=g \epsilon \beta d^{4} \kappa^{-1} \nu^{-1}$ (Rayleigh number), where $g$ is the acceleration due to gravity, $\epsilon$ the volume expansion coefficient, $\beta=-\partial T / \partial z$ the vertical temperature gradient, $\kappa$ the coefficient of thermal conductivity, and $\nu$ the coefficient of kinematic viscosity.
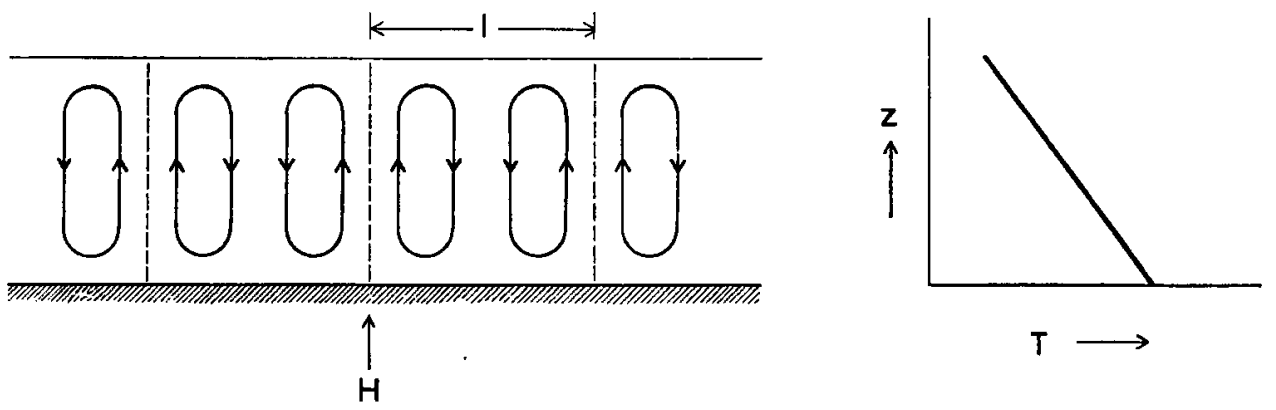

Fig. 14. Side view of thermally produced convection in a liquid heated below (left), and vertical distribution of temperature (right). 
The critical Rayleigh number at which the convection begins is about 1700 if both the top and bottom boundaries are rigid. It is also known that the characteristic horizontal size of the convection cell $l$ is about $3 d$.

In applying Rayleigh's criterion to meteorological problems, we must consider the effect of the earth's rotation. To understand the effect of the Coriolis force on convection, a theorem derived by Taylor [1921] is quite useful. First, we shall consider the motion of an incompressible, homogeneous fluid in a rotating framework. The equation of motion for the fluid is given by

$$
(\partial \nabla / \partial t)+(\mathbf{v} \cdot \nabla) \mathbf{v}+2 \mathbf{\Omega} \times \nabla=-\alpha \nabla \mathbf{p}+\nabla \Phi+\nu \nabla^{2} \mathbf{v}
$$

where the notation is the same as that in (1.1), except that molecular viscosity is assumed here. Assuming homogeneous fluid, the vorticity equation (see equation $1.12 a$ ) becomes

$$
(\partial \omega / \partial t)-\nabla \times[\nabla \times(\omega+2 \Omega)]=\nu \nabla^{2} \omega
$$

where $\omega=\nabla \times \nabla$ is the relative vorticity. If, in addition, we assume that the motion is slow and steady and that the viscosity is small,

$$
\nabla \times[\nabla \times \Omega]=0
$$

From (3.3) and the continuity equation $\nabla \cdot \nabla=0$,

$$
(\boldsymbol{\Omega} \cdot \nabla) \nabla=0
$$

This relation states that for slow steady motion in a uniformly rotating framework there is no velocity shear along the direction parallel to the axis of rotation. For $\Omega=(0,0, \Omega)$, equation 3.4 means that

$$
\partial u / \partial z=\partial v / \partial z=\partial w / \partial z=0
$$

Because we have the bottom boundary condition $w=0$, the motion becomes completely two dimensional. Even in the event that the fluid is nonhomogeneous, viscous, and possessing a thermal gradient, many features of convection in a rotating fluid can still be interpreted with this theorem.

Chandrasekhar [1953] has developed a theory of thermal convection with rotation and has found a relationship between the critical Rayleigh number and the angular velocity of the rotation. When the angular velocity is increased, the critical Rayleigh number becomes large and asymptotically tends to

$$
R_{a c} \rightarrow T_{a}{ }^{2 / 3}
$$

where $T_{a}=4 \Omega^{2} L^{4} \nu^{-2}$ (the Taylor number) is a measure of the ratio between Coriolis and viscous forces. $L$ is a characteristic length parameter. He found also a relation between the size of cells $l$ and $T_{a}$,

$$
l \rightarrow T_{a}^{-1 / 8}
$$

With increasing rotation, the required temperature gradient for onset of convection increases and the horizontal cell size decreases. The stronger the Coriolis force, the more the flow tends to be two dimensional. As the horizontal motion does not contribute to the heat release, convection will be suppressed unless a 
stronger temperature decrease with height is applied to the fluid. The shrinking of the cell dimensions is the result of the self-adjustment of fluid motion to avoid loss of kinetic energy of horizontal motion. The smaller cell size means that the cell pattern is elongated vertically and serves to transport heat more efficiently. Experimental verification of this theory has been undertaken by Nakagawa and Frenzen [1955].

Let us return to the problem of tropical cyclones. The problem is, in short, to reduce the tropical cyclone to Bénard cell convection and discuss the possibility of thermal instability as an explanation of tropical cyclone formation.

In the atmosphere, the variation of pressure with height is so large that we must consider the temperature change due to adiabatic compression and expansion. Therefore, in order to describe the stability of the atmosphere, we must use the vertical gradient of potential temperature (see equation 1.23) instead of temperature itself. Except on certain occasions in the surface layer, the gradient of potential temperature $\partial \Theta / \partial z$ is usually positive. Therefore pure (dry) thermal convection is unlikely to explain the tropical cyclone formation.

However, once condensation has taken place in the ascending air, liberation of the latent heat supplies additional buoyancy. The temperature change of the air is specified by the conservation of equivalent potential temperature (see equation 1.27)

$$
(d / d t) \ln \Theta_{\iota}=0
$$

If the vertical gradient of equivalent potential temperature is negative, the air is convectionally unstable. In Figure 15 the vertical distribution of the potential temperature and that of the equivalent potential temperature taken from Jordan's mean tropical atmosphere for the hurricane season are shown. In the lower half of the troposphere, we have a negative gradient of equivalent potential temperature.

Another consideration in theories of atmospheric convection, apart from those of the pure hydrodynamical case, is that we cannot assume the quasi-stationary

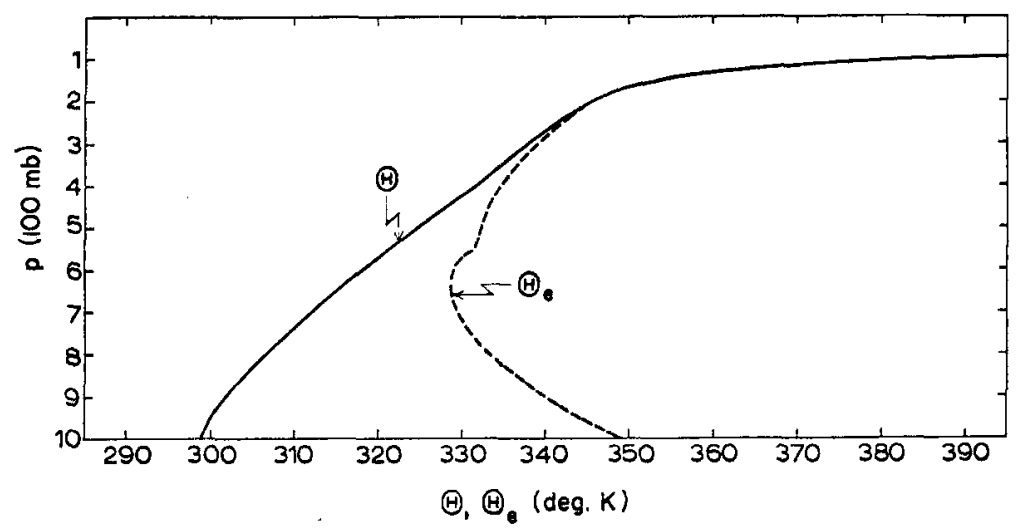

Fig. 15. Vertical distribution of potential temperature (solid line) and that of equivalent potential temperature (dashed line) for mean West Indies atmosphere during hurricane season (July-October) (based on Jordan's [1958a] data). 
onset of convection, because when condensation takes place in the latently unstable atmosphere the existing value of $\partial \Theta_{.} / \partial z$ usually exceeds the critical value expected from the concept of the critical Rayleigh number. Therefore, we must discuss the growth rate of convection given $\partial \Theta . / \partial z$ and find the maximum value of the growth rate as a function of the vertical and horizontal size of the convection.

Haque [1952], Kasahara [1952], and Syono [1953] first discussed the stability criteria of the tropical atmosphere by taking the negative vertical gradient of equivalent potential temperature into consideration. Recently the theory has been re-examined by Lilly [1960], Kuo [1961], and Yanai [1961b] for various distributions of $\Theta$, and by including the viscous dissipation. According to the most

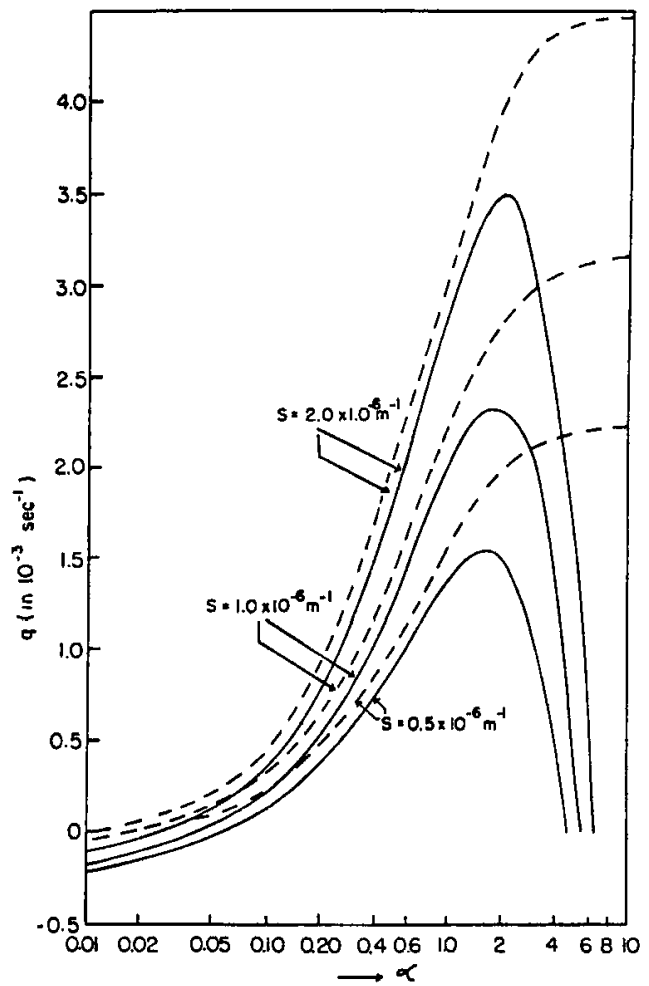

Fig. 16. Growth rate $q$ (in $10^{-8} \mathrm{sec}^{-1}$ ) of convection in a conditionally unstable atmosphere against wave number $\alpha$ for three different values of static stability factor $S$. Dashed curves give inviscid approximation; solid curves give eddy viscosity coefficient $\nu=10^{3} \mathrm{~m}^{2} \mathrm{sec}^{-1}$ [after Kuo, 1961]. complete treatment, by Kuo, the growth rate of convection (we assume an exponential time factor $\exp (\sigma t))$ in an unstably stratified atmosphere is given by

$$
\sigma=\sqrt{\frac{g \alpha^{2} S-k^{2} f}{1+\alpha^{2}}}-\left(\frac{\pi}{h}\right)^{2}\left(\alpha^{2}+k^{2}\right) \nu
$$

Here $S=-\partial \ln \Theta_{\theta} / \partial z$ is a measure of the unstable stratification, $\alpha$ and $k$ are the horizontal and vertical wave numbers (proportional to the reciprocal of cell size), respectively, $f$ is the Coriolis parameter, $h$ is the depth of the unstable layer, and $\nu$ is the eddy viscosity coefficient.

Figure 16 shows the relationship between the growth rate and the horizontal wave number $\alpha$. The larger the wave number or the smaller the horizontal cell size, the faster the convection will grow owing to the negative vertical gradient of $\Theta_{0}$. However, an extremely large wave number (or very small cell size) will meet a large dissipation effect due to viscosity, and the growth rate will drop at extremely large wave numbers. On the other hand, the Coriolis factor becomes important at smaller wave numbers (large cell sizes). There is a limiting wave number $\alpha_{0}$ which separates the amplifying smaller cells from the decaying larger cells.

In the earlier studies, this limiting wave number or cell size was identified with that of tropical cyclones. However, this interpretation is not suitable, because 
even with the convection having this limiting scale, smaller convections still will grow. The maximum growth rate, in a numerical example shown in Figure 16, corresponds to the wave number 1.6. This gives a corresponding horizontal scale of circular cell of $l=0.48 h$. As $h$ is several kilometers, this cell size will be that of cumulus convection.

Even if we adopt a more realistic distribution of the static stability factor $S$, the preferred scale does not change much. Thus the application of the thermal instability does not seem to be appropriate for tropical cyclone formation.

Numerical model researches. Several attempts have been made to simulate the process of tropical cyclone formation and development by integrating the hydrodynamical equations numerically with high-speed electronic computers. This method is unlike linear perturbation analysis in that we can include nonlinear interactions and more complicated distributions of various parameters. The nonlinear coupling of growing convection and the environment might overcome the difficulty which appeared in the thermal instability theories.

Kasahara [1961a] and Syono [1962] have tried to compute the growth of a large-scale vortex motion which is embedded in a conditionally unstable atmosphere. Initially the air was assumed to be at rest; then a radial circulation was introduced. By releasing latent heat of condensation, this circulation was intensified; through the Coriolis force the tangential motion also was started. However, as the computation went on, small-scale fluctuations appeared and the cell-like convective motions grew enormously. Growth of the large-scale circulation, initially expected, was not produced. Thus the same difficulty involved in linear perturbation theories was encountered.

The real tropical cyclone seems to possess the combined convection characteristics of both the large-scale motion and the numerous cumulus clouds. These two different convective motions do not seem to compete with each other; they may interact mutually and maintain an equilibrium state. How can the energy partition between these two scales be realized in the atmosphere? This is one of the most serious questions that lead many researchers to try to find a better model of the tropical cyclone.

There have been several suggested explanations of the energy partition between the tropical cyclone and the embedded cumulus clouds. Once the cumulus convection due to condensation starts, it will reach the fully developed stage very quickly, because of its large growth rate. The upward heat transport by the convection may change the thermal stratification of the environment. A final fully turbulent but balanced stage will then be realized. In this stage, a deep neutral layer may develop. $K u o[1962 a]$ has proposed a nonlinear eddy diffusion process which suppresses the enormous growth of thermal convection and assures an equilibrium stage.

Hypothesis of development of large-scale vortex motion. Difficulties encountered in the thermal buoyant instability theory were essentially the result of the assumption of negative static stability. Under this hypothesis, the preferred scale of perturbation becomes very small. The preferred scale obtained is that of cumulus convection. In reality, we observe numerous cloud towers in the tropical cyclone. These cloud towers must be driven by buoyancy. At the same time, we know that these cloud towers cover only a small part of the large area of the tropical cyclone. 
Over the remaining area the condensation process does not take place; there the static stability factor should be considered as positive when referred to unsaturated motion.

It seems reasonable to postulate that the stratification of the tropical atmosphere, when we average over a wide area, is stable insofar as large-scale motion is concerned. Then we may be able to parameterize the role of cumulus clouds as heat sources in this average-stability atmosphere. Later we shall examine the parameterization problem further. Meanwhile, we shall assume this stable model and shall try to find a mechanism to explain the growth of large vortex motion.

Historically, Sawyer [1947] first advocated the so-called inertial instability theory of tropical cyclone formation. To explain the organization of small buoyant convection into a large-scale motion, he considered that absolute vorticity in the upper troposphere may become negative at certain times and that this causes a divergent outflow in the upper layers; then it may serve to organize the buoyant convection into a large-scale vortex. Kleinschmidt [1951] also considered the inertial instability as the leading mechanism for tropical cyclone formation but in a slightly different manner from Sawyer's consideration. In his model, the inertial instability was considered along the surfaces of equal equivalent potential temperature which were supposed to be inclined vertically. Thus the inertial instability was considered to be a result of the warm-core structure.

To discuss these instability mechanisms systematically, we must return to a classical problem, i.e., the stability properties of an atmospheric air ring. There have been several papers on this problem [see Hoiland, 1941; Eliassen, 1949; Fjørtoft, 1950].

We shall consider a circular vortex ring of air which can be identified with an incipient tropical cyclone. As the basic state, we assume a balanced motion expressed by the gradient wind relation and the hydrostatic equilibrium. For simplicity, we introduce the pressure coordinate system [Eliassen, 1949]. Then

$$
\begin{aligned}
f \bar{v}_{\lambda}+\left(\bar{v}_{\lambda}{ }^{2} / r\right) & =(\partial \bar{\Phi} / \partial r)_{\nu} \\
-\bar{\alpha} & =\partial \bar{\Phi} / \partial p
\end{aligned}
$$

where $f$ is the Coriolis parameter, and $\bar{\nu}_{\lambda}, \bar{\Phi}$, and $\bar{\alpha}$ are the tangential velocity, the geopotential, and the specific volume of the air in the basic state. The two equations can be reduced to the thermal wind equation (cf. equation 1.8)

$$
\left(f+\frac{2 \bar{v}_{\lambda}}{r}\right) \frac{\partial \bar{\nu}_{\lambda}}{\partial p}=\frac{\partial^{2} \bar{\Phi}}{\partial r \partial p}=-\left(\frac{\partial \bar{\alpha}}{\partial r}\right)_{\nu}
$$

The baroclinicity is simply expressed by the lateral gradient of specific volume along the isobaric surface.

Now we shall consider an axially symmetric perturbation superimposed on the vortex. Perturbation quantities are tangential velocity $v_{\lambda}^{\prime}$, radial velocity $v_{r}^{\prime}$, vertical $p$ velocity $\omega^{\prime}=d p / d t\left(\omega^{\prime}<0\right.$, upward motion), geopotential $\Phi^{\prime}$, and specific volume $\alpha^{\prime}$. Perturbation equations then are:

$$
\begin{aligned}
\left(\partial v_{\lambda}{ }^{\prime} / \partial t\right)+\eta v_{r}^{\prime}+\left(\partial \bar{v}_{\lambda} / \partial p\right) \omega^{\prime} & =F_{\lambda} \\
\left(\partial v_{+}^{\prime} / \partial t\right)-\xi v_{\lambda}^{\prime}+\left(\partial \Phi^{\prime} / \partial r\right) & =0
\end{aligned}
$$




$$
\begin{gathered}
\left(\partial \Phi^{\prime} / \partial p\right)+\alpha^{\prime}=0 \\
\left(\partial v_{r}^{\prime} / \partial r\right)+\left(v_{r}^{\prime} / r\right)+\left(\partial \omega^{\prime} / \partial p\right)=0 \\
\left(\partial \alpha^{\prime} / \partial t\right)-B v_{r}^{\prime}-S \omega^{\prime}=H
\end{gathered}
$$

where we simply assumed a tangential friction $F_{\lambda}$ and a heating function $H$. For the time being we shall assume these two to be independent outer forcing functions. Other notations are

$$
\eta=\left(f+\frac{\partial \vec{v}_{\lambda}}{\partial r}+\frac{\bar{v}_{\lambda}}{r}\right)_{p} \quad \xi=f+\frac{2 \tilde{v}_{\lambda}}{r} \quad B=\xi \frac{\partial \bar{v}_{\lambda}}{\partial p}=-\left(\frac{\partial \bar{\alpha}}{\partial r}\right)_{p}
$$

the baroclinicity, and $S=-(\bar{\alpha} / \bar{\Theta})(\partial \bar{\Theta} / \partial p)$ the static stability factor.

The continuity equation 3.15 is satisfied by using the Stokes stream function $\psi$ through which the radial velocity $v_{r}^{\prime}$ and the vertical $p$ velocity $\omega^{\prime}$ are related by

$$
v_{r}^{\prime}=\frac{1}{r} \frac{\partial(r \psi)}{\partial p} \quad \omega^{\prime}=-\frac{1}{r}\left\{\frac{\partial}{\partial r}(r \psi)\right\}_{D}
$$

Inserting the relation 3.17 into (3.13) and (3.16), and eliminating $\phi^{\prime}$ by using $\alpha^{\prime}=-\partial \phi^{\prime} / \partial p$, we obtain

$$
\frac{\partial^{2}}{\partial t^{2}}\left(\frac{\partial^{2} \psi}{\partial p^{2}}\right)-\xi \frac{\partial^{2} v_{\lambda}{ }^{\prime}}{\partial t \partial p}-B \frac{\partial^{2} \psi}{\partial r \partial p}+S\left(\frac{\partial^{2} \psi}{\partial r^{2}}+\frac{1}{r} \frac{\partial \psi}{\partial r}-\frac{\psi}{r^{2}}\right)_{p}=\left(\frac{\partial H}{\partial r}\right)_{\nu}
$$

From (3.12),

$$
\xi \frac{\partial^{2} v_{\lambda}^{\prime}}{\partial t \partial p}+\xi \eta \frac{\partial^{2} \psi}{\partial p^{2}}-B \frac{\partial}{\partial p}\left(\frac{\partial \psi}{\partial r}+\frac{\psi}{r}\right)_{p}=\xi \frac{\partial F_{\lambda}}{\partial p}
$$

From (3.18) and (3.19), we can eliminate $v_{\lambda}^{\prime}$ and finally obtain an equation for the stream function:

$$
\left(\frac{\partial^{2}}{\partial t^{2}}+\xi \eta\right) \frac{\partial^{2} \psi}{\partial p^{2}}-B\left(2 \frac{\partial^{2} \psi}{\partial r \frac{\partial}{\partial p}}+\frac{1}{r} \frac{\partial \psi}{\partial p}\right)+S\left(\frac{\partial^{2} \psi}{\partial r^{2}}+\frac{1}{r} \frac{\partial \psi}{\partial r}-\frac{\psi}{r^{2}}\right)_{D}=\left(\frac{\partial H}{\partial r}\right)_{D}+\xi \frac{\partial F_{\lambda}}{\partial p}
$$

Equation 3.20 has three stability coefficients; the inertial stability factor

$$
\xi \eta=\left(f+\frac{2 \bar{v}_{\lambda}}{r}\right)\left(f+\frac{\partial \bar{v}_{\lambda}}{\partial r}+\frac{\bar{v}_{\lambda}}{r}\right)_{z}
$$

the baroclinicity

$$
B=-(\partial \bar{\alpha} / \partial r)_{p}
$$

and the static stability

$$
S=-\bar{\alpha}(\partial \ln \bar{\Theta} / \partial p)
$$

The radial gradient of $H$ and the vertical gradient of $F_{\lambda}$ act as the forcing functions.

Next we consider the boundary condition. Let us assume that the stream lines are within a finite domain of $r-p$ space and assume further that $\psi=0$ along the boundary (Figure 17). 
Two types of motion can be described by (3.20). Let us call these two the 'balanced' motion and the 'unbalanced' motion.

The balanced motion: If we can assume that the gradient wind relation is always satisfied even for the perturbation, we can drop the radial acceleration $\partial v_{r}^{\prime} / \partial t$ from (3.13). Then the time derivative in (3.20) also vanishes. The resulting equation

$\xi \eta \frac{\partial^{2} \psi}{\partial p^{2}}-B\left(2 \frac{\partial^{2} \psi}{\partial r \partial p}+\frac{1}{r} \frac{\partial \psi}{\partial p}\right)+S\left(\frac{\partial^{2} \psi}{\partial r^{2}}+\frac{1}{r} \frac{\partial \psi}{\partial r}-\frac{\psi}{r^{2}}\right)_{p}=\left(\frac{\partial H}{\partial r}\right)_{p}+\xi \frac{\partial F_{\lambda}}{\partial p}$

becomes a diagnostic equation for the stream function under the given forcing function and stability coefficients. The heating function may decrease with increas-

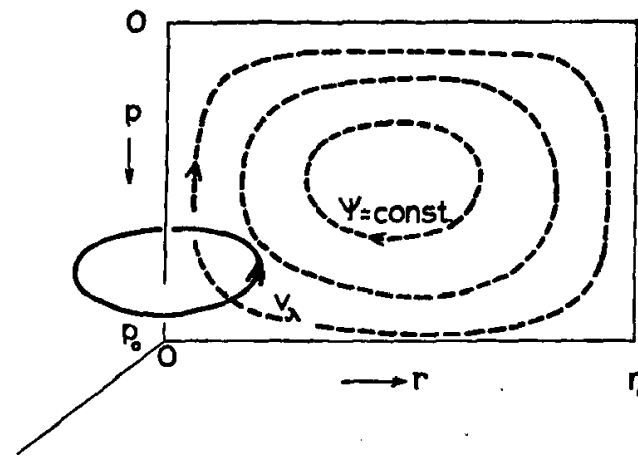

Fig. 17. Schematic view of vortex and superimposed radial-vertical circulation. ing radius, and the friction decreases with increasing height; thus the total forcing function should be negative. Equation 3.21 has the form of a generalized Poisson equation when the ellipticity condition

$$
B^{2}-\xi \eta S<0
$$

is satisfied. The form of (3.21) is similar to that of the equation describing suspension equilibrium of an elastic membrane with a load. Because of the boundary condition $\psi=0$, we can get the distribution of $\psi$ as schematically given in Figure 17. Eliassen

[1952] has discussed the applicability of this sort of thermally and frictionally controlled balanced circulation to the atmospheric general circulation problem and later applied this concept to the vertical circulation around the meteorological front [Eliassen, 1959]. Estoque [1962] has applied a similar equation for the vertical circulation within the tropical cyclone.

One of the difficulties involved in this concept is that the validity of (3.21) is restricted by the ellipticity condition 3.22. Thus under strong baroclinicity or negative absolute vorticity, the condition is violated and balanced circulation cannot exist. Unless we further formulate the time dependence of the forcing function, we do not get the explicit growth rate of the system, and there is no special interpretation on the preferred scale of the system.

The unbalanced motion: The second kind of motion described by (3.20) is characterized by existence of $\partial v_{r}^{\prime} / \partial t$. When we admit the existence of $\partial v_{r}^{\prime} / \partial t$, the system can have an explicit growth rate under certain conditions. For simplicity, we shall assume an exponential growth $\partial \psi / \partial t=\psi e^{\sigma t}$, where $\sigma$ is the growth rate of the system. Then (3.20) becomes

$$
\left(\sigma^{2}+\xi \eta\right) \frac{\partial^{2} \psi}{\partial p^{2}}-B\left(2 \frac{\partial^{2} \psi}{\partial r \partial p}+\frac{1}{r} \frac{\partial \psi}{\partial p}\right)+S\left(\frac{\partial^{2} \psi}{\partial r^{2}}+\frac{1}{r} \frac{\partial \psi}{\partial r}-\frac{\psi}{r^{2}}\right)_{p}=\left(\frac{\partial H}{\partial r}\right)_{p}+\xi \frac{\partial F_{\lambda}}{\partial p}
$$


General treatment of (3.23) is difficult. However, when we have a condition

$$
B^{2}-\left(\sigma^{2}+\xi \eta\right) S>0
$$

the homogeneous part of (3.23) may have even solutions in the $r-p$ plane satisfying the boundary condition $\psi=0$. As we have assumed a stable stratification, (3.24) is equivalent to

$$
B^{2} / S-\xi \eta>\sigma^{2}>0
$$

This inequality shows that the growth of this type of free convection is possible only when the baroclinicity is large enough or the inertial stability factor $\xi \eta$ is negative.

The requirement $B^{2} / S-\xi \eta>0$ has a simple physical meaning. By rewriting $B$ and $S$ using potential temperature, and by rewriting the inertial stability factor relating to the absolute angular momentum $A=r\left(\bar{v}_{\lambda}+\frac{1}{2} f r\right)$, we can show that the inequality 3.25 is equivalent to

$$
\tan \theta>\tan \lambda
$$

where $\theta$ is the angle between the isentropic surface and the horizontal plane, and $\lambda$ is the slope of the surface of equal absolute angular momentum. The meaning of (3.26) can be seen in Figure 18. Suppose an air particle moves along an isentrope with constant angular momentum $\left(p \rightarrow p^{\prime}\right)$; then the particle will be

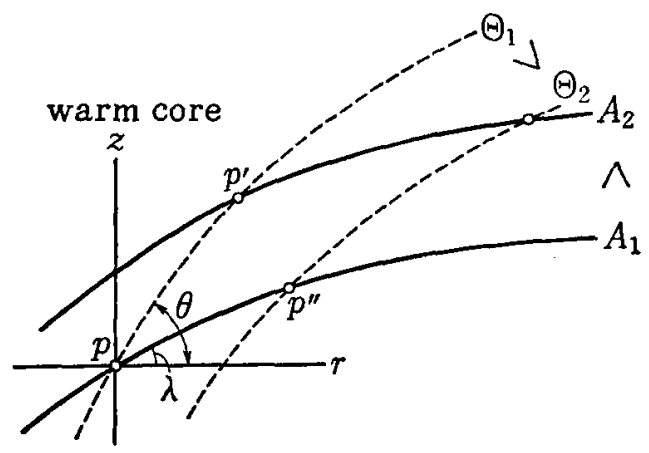

Fig. 18. Inertial instability condition in baroclinic vortex. Solid curves are isolines of absolute angular momentum $A$. Dashed curves are those of (equivalent) potential temperature. accelerated outward by the excess of centrifugal force. When the air particle moves along a line of equal absolute angular momentum with constant potential temperature $\left(p \rightarrow p^{\prime \prime}\right)$, the particle must gain buoyancy because it possesses a higher potential temperature than the surroundings. Thus (3.26) can also be written

$(\partial \Theta / \partial r)_{\Delta}<0$ or $\left(\partial A^{2} / \partial r\right)_{\theta}<0$

This formulation expresses baroclinic instability along a constant absolute angular momentum surface, or inertial instability along a constant potential temperature surface.

Kleinschmidt [1951] first mentioned an instability of this type while explaining the relatively large horizontal scale of the tropical cyclone. Kuo $[1954,1956]$ has discussed the instability in relation to the interpretation of results obtained in so-called rotating dishpan experiments. The criterion 3.25 has the same meaning as Kuo's criterion for onset of free meridional circulation (upper Hadley regime) observed in the experiment. ${ }^{3}$

- Fultz [1956, 1959] and Hide [1953, 1958] have made extensive experimental studies on the behavior of fluid contained in a rotating disk or an annular cylinder under the radial temperature contrast. There are three characteristic regimes of fluid motion according to the combination 
As a special case, in a completely barotropic atmosphere $(B=0)$, (3.25) is reduced to

$$
\left(f+\frac{2 \bar{v}_{\lambda}}{r}\right)\left(f+\frac{\partial \bar{v}_{\lambda}}{\partial r}+\frac{\bar{v}_{\lambda}}{r}\right)_{p}<0
$$

This is the criterion of pure inertial instability advocated by Sawyer [1947] and recently by Alaka [1958, 1961, 1962, 1963].

It should be mentioned that criterion 3.25 is necessary but is not sufficient for instability. The same statement holds for (3.28) for the barotropic case. To get a necessary and sufficient condition of instability, we must solve (3.23) and derive the growth rate as a function of horizontal and vertical scales of the radialvertical circulation. For the barotropic case the growth rate is readily given by

$$
\sigma=\sqrt{-\xi \eta-\left(b_{\iota} p_{0} / n \pi r_{0}\right)^{2} S}
$$

where $b_{l}$ is the horizontal wave number given by a root of first-order Bessel function, $n$ is the vertical wave number given by an integer, and $p_{0}$ and $r_{0}$ are the surface pressure and outer radius of the vortex. As the stable stratification acts to suppress the vertical displacement of air, it is natural that the first horizontal mode of the solution, $b_{l}=b_{1}$, grows most rapidly. In the assumed nonviscous air, we will select the vertical mode such that a larger $n$ will give a larger growth rate. However, suitable selection of eddy viscosity will suppress the growth of extremely thin multiple circulations. If we justify the selection of $n=1$ by a suitable viscous effect, the minimum critical value of $-\xi \eta$ required for instability is

$$
(-\xi \eta)_{\min }=\left(b_{1} p_{0} / \pi r_{0}\right)^{2} S
$$

This requires an enormously large negative inertial stability factor which is not observed in the usual situation.

Analytical solution of (3.23) including the baroclinicity term is difficult because the separation of variables does not fit the boundary curve. Kasahara [1961b] and Yanai [1961b] have attempted to obtain an approximate solution by altering the boundary. The results contradict each other. We may need more precise numerical evaluation of the problem. However, as may be suggested by the preceding argument on the air particle movement, there must be a preferred scale for the circulation pattern because to be accelerated, the air particle must choose a vertically inclined path between the isentrope and the line of equal absolute angular momentum.

Recent progress in theoretical studies. No development mechanism of the

of the thermal Rossby number $R_{O T^{*}} \sim a T / \partial r\left(/ \Omega^{2}\right)$, which is the ratio of the radial temperature gradient to the square of the rate of rotation, and $G^{*} \sim g / \Omega^{2}$, the ratio of the gravity to centrifugal acceleration.

1. The lower Hadley symmetric regime: A circular symmetric flow with a slow meridional circulation appears at low $R_{O T^{*}}$ and high $G^{*}$ (slow rotation).

2. The Rossby wave regime: Wave disturbances propagate around the rotating axis at moderate value of $R_{O T^{*}}$ and low $G^{*}$ (high rotation). The wave number decreases with increase of $R_{O T^{*}}$ at constant $G^{*}$.

3. The upper Hadley symmetric regime: Flow pattern again becomes symmetric at very high $R_{O T}{ }^{*}$ and high $G^{*}$. 
tropical cyclone has yet found general acceptance. However, there is broad agreement among specialists. First of all, it is generally recognized that the tropical cyclone itself and the cumulus convection embedded within the cyclone are driven by different mechanisms. Then, the current tendency of theoretical study is to discuss these two convections separately and to reconstruct the model of the whole system by considering the interaction between the large-scale cyclone and the small-scale cumulus clouds. Many researchers have been involved in the problem of how to parameterize the small-scale clouds in the dynamical framework and how to maintain the equilibrium between two different convections.

Charney et al. [1964], Estoque [1964], Kasahara [1964], Kuo [1964], Ooyama [1964], Rosenthal [1964], and Syōno and Hirota [1963] have proposed a model or an idea of construction for the tropical cyclone. Though there are technical diffrences, the basic concepts are, as stated above, very similar.

Opinion is split, however, on the interpretation of the growth of the large-scale system itself. Neither the theory of balanced circulation controlled by differential heating and friction nor that of inertial instability which is an essential result of unbalanced model has yet met with complete acceptance. One of the criticisms against the inertial instability hypothesis (along the isentropes) is that in real tropical cyclones we do not find the slope of the isentropic surfaces so strongly inclined [see Alaka, 1963]. However, this depends only on the interpretation of the conservative thermodynamic property within the tropical cyclone. Kleinschmidt originally considered that equivalent potential temperature was the conservative property. As real atmosphere is a mixture of saturated and unsaturated airs, we inevitably need some kind of averaging process in theoretical treatment. If we express the heating process due to cumulus-scale convection by suitable parameterization, the horizontally averaged static stability may remain positive but will be very much reduced from the pure dry adiabatic static stability. Later on, we will define an effective static stability which will lead to modification of the definition of isentropes.

\section{A TENTATIVE MODEL OF TROPICAL CYCLONE FORMATION}

From the discussion so far presented, it is evident that there are many problems remaining to be solved before a complete understanding of tropical cyclone formation is obtained. During the past few years study has been focused on several crucial questions. It may be worth while to try to construct a most plausible model of the formation based on current knowledge.

The whole process of the formation is, of course, a continuous series of several events. However, for a clear statement of the problems, let us consider the three major phases of the formation as defined in section 2 : the wave stage, the warming stage, and the developing stage.

The wave stage. First we must consider the easterly waves with high-level anticyclones as the potential embryo of the tropical cyclone. According to my preliminary analysis in the Pacific region, even ordinary or weak wave disturbances are associated with systematic organization of relative vorticity, horizontal divergence, and vertical motion. The vertical motion associated with the waves has a re- 
markable controlling effect on the upper-air temperature and the shower activity [Yanai, 1964].

The structure and dynamics of the easterly waves and the upper-level large anticyclones have not been fully understood. The puzzling question raised by Riehl's finding of cold-core structure has not yet been conclusively answered. As the upward motion associated with the easterly wave is taking place in relatively colder air, the kinetic energy release cannot come from local conversion of potential energy. We have to seek for other possibilities of kinetic energy maintenance either from the pressure work from an outer region or from the supply of kinetic energy of the basic easterly current. In my opinion, the horizontal shear in the easterlies (Figure 19) may have something to do with momentum transport and conversion of kinetic energy from the basic current to the disturbance. However, for any definite statement on this matter, we need a quantitative investigation of energetics of easterly waves and probably a climatological study of the relationship between the wave activity and the intensity of the trade easterlies.

Concerning several characteristics of the easterly waves, Rosenthal [1960] has developed a linear theory in which the distributions of stream lines and horj-
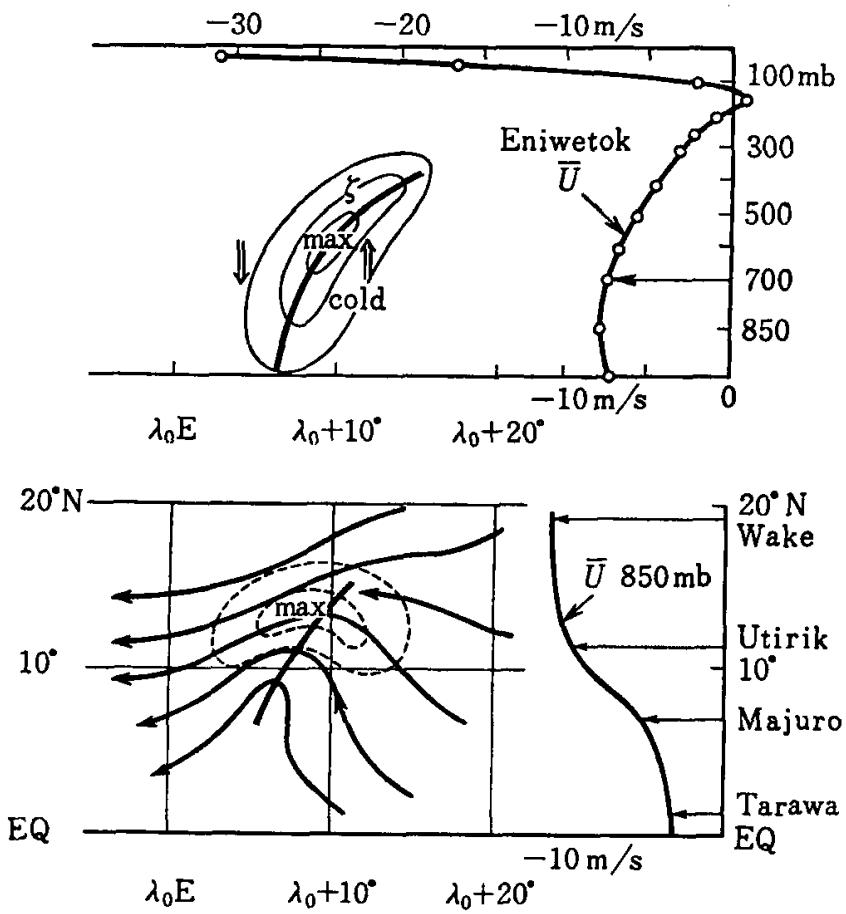

Fig. 19. Model of easterly wave. Top: Side view of easterly wave (left) and vertical distribution of basic current (right). On the left, thin lines are isolines of vorticity. The sign of vertical motion is shown by arrows. Bottom: Horizontal view of easterly wave (left) and latitudinal distribution of basic current (right). On the left, stream lines (solid arrows) and isotachs (thin dotted lines) are shown. 
zontal divergence are obtained. The main factor determining the horizontal distributions of these characteristics was the latitudinal variation of the basic easterly current. On the other hand, Yanai [1961b] has made a simple estimation of the distribution of the vertical motion associated with the easterly wave. Assuming the quasi-balanced equations of motion, the upward motion behind the wave axis and the downward motion in front of the axis were obtained. The main cause of the vertical motion was attributed to a dynamical effect from the differential advection of vorticity in a vertically shearing easterly current (Figure 19).

Considering very weak baroclinicity pre-existing in the tropical atmosphere, the dynamically induced vertical motion causes a cooling in the troposphere before the condensation process becomes significant. The disturbance is therefore baroclinically stable, and only a very slow growth may be made possible by the supply of kinetic energy from the basic current.

Two factors may favor the growth of the wave disturbance. A migratory high-level anticyclone sometimes becomes superimposed on the low-level wave. The weakening of the inertial stability factor may cause intensification of the pre-existing vertical motion. At the same time, if the wave system is in the region of moist oceanic air, the intensified vertical motion creates a thick moist layer. Thus the cumulus convection will be promoted within the synoptic scale upward motion. The release of latent heat of condensation will gradually modify the temperature structure of the disturbance. A numerical model study of the development of an easterly wave was attempted by Berkofsky [1960].

The warming stage. The transformation of a cold-core disturbance to a warm-core one is the most amazing phase of tropical cyclone formation. It is certain that the major factor in this warming process is the release of latent heat of condensation.

Yanai [1961a, 1963] has pointed out two interesting problems observed during the warm-core creation of typhoon Doris of 1958. The first is that the warming was a very slow process during which none of the characteristics of pre-existing disturbances, such as the large-scale vertical motion and surface pressure, showed any abrupt change. Thermodynamic analysis has shown that, during the creation of the warm core the warming effect due to the condensation and the cooling effect due to the ascending motion were nearly balanced. Only a slight imbalance between these two effects resulted in the final warming of the core region. The second point is that the first formation of the warm core took place in the layer between 300 and $400 \mathrm{mb}$, then the warming expanded downward. Colon et al. [1961] have found a similar occurrence in hurricane Daisy of 1958.

If we apply the thermally buoyant instability concept directly to the largescale vertical motion, the time change of thermal structure will be entirely dependent on the vertical gradient of equivalent potential temperature. As we have a negative gradient of equivalent potential temperature in the lower troposphere, the result will be warming from below. The feedback of released energy to the vertical motion must also produce a more abrupt change in the characteristics of the pre-existing circulation system.

The implications of these are as follows. As has been indicated in several theoretical analyses, the release of buoyant energy cannot be directly associated 
with large-scale vertical circulation, but with small-scale cumulus convection. Therefore the release of the latent heat of condensation is not explicitly related to the observed macroscopical upward motion. In order to explain the peculiar features of the warming process, it seems necessary to separate the motion into two parts, i.e., that of the tropical cyclone itself and that of the cumulus clouds embedded within.

When we consider the difference in the scales of these two types of motion both in space and in time, it is not plausible to treat both phenomena simultaneously. During the rather slow development of a tropical cyclone, the cumulus convection goes through various phases of its own life cycle whose time scale may be $30 \sim 60$ minutes. New clouds form while older convective clouds die. Consequently we must consider the statistical average of the total cloud activity both in time and in space.

We shall consider the problem of parameterization of the heating function due to these clouds. Formally, the thermodynamic equation for the large-scale system can be written as

$$
\frac{\partial \bar{\Theta}}{\partial t}+\nabla_{h}(\overline{\Theta v})+\frac{\partial}{\partial p}(\overline{\Theta \omega})=\frac{1}{c_{p}}\left(\frac{p_{0}}{p}\right)^{(\gamma-1) / \gamma} \frac{d \bar{h}}{d t}
$$

where the overbar denotes the average horizontally in space and in time. A suitable averaging scale may be chosen with the viewpoint of resolution for the description of a tropical cyclone development. Since there is no special evidence suggesting a significant horizontal advection of heat by eddies, the above equation can be transformed to

$$
\frac{\partial \bar{\Theta}}{\partial t}+\nabla_{h} \cdot(\bar{\Theta} \bar{v})+\frac{\partial}{\partial p}(\bar{\Theta} \bar{\omega})+\frac{\partial}{\partial p}\left(\overline{\Theta^{\prime} \omega^{\prime}}\right)=\frac{1}{c_{p}}\left(\frac{p_{0}}{p}\right)^{(\gamma-1) / \gamma} \frac{d \bar{h}}{d t}
$$

In (4.2), besides the heating term due to the condensation on the right, we have an eddy vertical transport term due to the correlation between the fluctuations of temperature and vertical velocity. This kind of formulation was first discussed by Priestley and Swinbank [1947] for other purposes.

Our aim is to obtain an approximate idea of how the increase of the average temperature is explained by the release of condensation and by the eddy vertical transport of heat by cloud-scale convection.

A vertical heat transport mechanism directly related to cumulus towers has been advanced by Riehl and Malkus [1958]. They studied the heat budget over the equatorial trough zone and found that concentrated upward heat transport due to active cumulus clouds is important to maintain the high heat content of the upper troposphere. They have further developed this 'hot tower' hypothesis into a budget study of heat in the central core region of a hurricane [Riehl and Malkus, 1961].

Here we shall consider a similar mechanism in order to explain several features of the warming process in the tropical cyclone formation. For a further specification of each term of (4.2), we shall assume an extremely simplified model of atmosphere which contains cumulus towers. The area ratio of cloud towers to the total area is assumed to be a slow varying parameter in space.

We define the vertical motion $(d p / d t)$ and potential temperature in convective 
clouds by $\omega_{0}, \Theta_{0}$ and those in the remaining area as $\bar{\omega}$ and $\widetilde{\Theta}$, respectively. By definition,

$$
\begin{aligned}
\bar{\Theta} & =a \Theta_{0}+(1-a) \tilde{\Theta}=a \Theta^{*}+\tilde{\Theta} \\
\bar{\omega} & =a \omega_{0}+(1-a) \bar{\omega}=a \omega^{*}+\bar{\omega} \\
\bar{\Theta} \omega & =a \Theta_{0} \omega_{0}+(1-a) \tilde{\Theta} \bar{\omega}=a \Theta^{*} \omega^{*}+a\left(\tilde{\omega} \Theta^{*}+\omega^{*} \tilde{\Theta}\right)+\tilde{\Theta} \bar{\omega}
\end{aligned}
$$

Here $\Theta^{*}=\Theta_{0}-\widetilde{\Theta}$ and $\omega^{*}=\omega_{0}-\bar{\omega}$ are the excess potential temperature and the excess vertical $p$ velocity in clouds, respectively, and $a$ is the area ratio of cloud towers to the whole space.

We thus obtain an expression for the eddy heat transport term:

$$
\overline{\Theta^{\prime} \omega^{\prime}}=\overline{\Theta \omega}-\bar{\Theta} \bar{\omega}=a(1-a) \Theta^{*} \omega^{*}
$$

Then the thermodynamic equation for the large-scale system becomes

$$
\frac{\partial \bar{\Theta}}{\partial t}+\bar{v}_{k} \cdot \nabla_{h} \bar{\Theta}+\bar{S} \bar{\omega}+a(1-a) \frac{\partial}{\partial p}\left(\Theta^{*} \omega^{*}\right)+a(1-a) \frac{L}{c_{p}}\left(\frac{p_{0}}{p}\right)^{(\gamma-1) / \gamma} \frac{\partial q_{z}}{\partial p} \omega^{*}=0
$$

where

$$
\bar{S}=a\left(\frac{\partial \Theta_{a}}{\partial p}\right)_{0}+(1-a) \frac{\partial \tilde{\Theta}}{\partial p}
$$

is the newly defined effective static stability for the average atmosphere.

Equation 4.5 suggests that the existence of small-scale convective clouds reduces the mean static stability factor and at the same time contributes to the transport of heat if the clouds are warmer than the surroundings and the upward velocity within the clouds is larger than that of the surroundings. Not only the local release of latent heat of condensation, but also the vertical transport of this sensible heat may be quite important to the warming of the upper tropospheric air.

When we take limiting cases $a \rightarrow 0$ and $a \rightarrow 1$, equation 4.5 becomes

$$
(\partial \bar{\Theta} / \partial t)+\bar{v} \cdot \nabla \bar{\Theta}+\bar{S} \bar{\omega}=0 \quad \bar{S} \rightarrow \partial \Theta / \partial p
$$

and

$$
(\partial \bar{\Theta} / \partial t)+\bar{v} \cdot \nabla \bar{\Theta}+\bar{S} \bar{\omega}=0 \quad \bar{S} \rightarrow \partial \Theta_{0} / \partial p
$$

respectively. The first is the pure dry adiabatic equation, and the second corresponds to the equation valid for fully saturated air.

We shall discuss the warming process qualitatively on the basis of (4.5). At the beginning, the synoptic scale vertical motion exists within a disturbance. As was discussed previously, the cold-core structure of a pre-existing disturbance will be explained by a forced ascent without having much condensation $(a \rightarrow 0)$. The temperature change will be approximately described by a dry adiabatic equation. The upward motion will cause relative cooling of the air (see Figure 20a). However, when cumulus convection starts within the broad ascending area (Figure $20 b$ ), the release of latent heat of condensation will modify the temperature within individual clouds. The vertical temperature distribution within clouds may come rapidly to a stage at which the equivalent potential temperature is nearly constant. 
This raises the average temperature. More important, the excess of temperature and that of upward velocity within clouds creates a vertical heat transport. Because the maximum excess of temperature is expected at around $400 \mathrm{mb}$, the level of maximum upward velocity may be at some level higher than this. The net heat transport which is the product of the excess potential temperature and the excess vertical velocity will have a maximum at about $400 \mathrm{mb}$. Thus a heat flux divergence causes a cooling effect in the lower layer, and a heat flux convergence will cause a warming in the upper layer. Figure $20 \mathrm{c}$ illustrates this effect. Combining the general

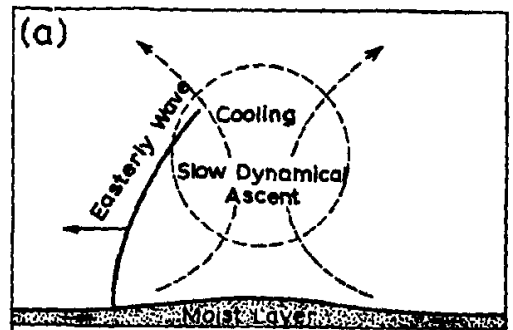

(b)

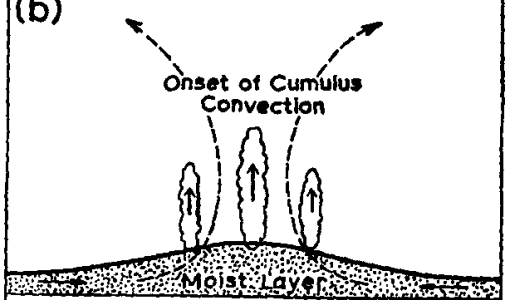

(c)

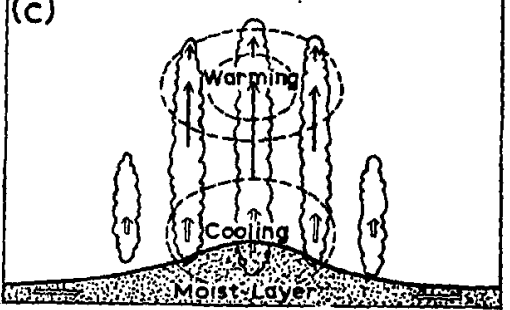

Fig. 20. Postulated change of thermal structure of a formative tropical cyclone due to cumulus convection. cooling effect by the large-scale ascent and the heat flux due to cumulus convection by assuming a certain area ratio $a$, we will be able to describe the gradual warming observed in the tropical cyclone formation.

According to Malkus et al. [1961], the area occupied by towering cumulonimbus is a small per cent of the area even in the core region of a mature hurricane (see Figure 21). Thus the effective static stability $\bar{S}$ may never become negative. Nevertheless, large upward velocity is concentrated in these clouds; thus warming may be achieved by the vertical heat transport in the cloud towers even in the average-stability atmosphere.

For a more definite specification, we need a model of elements of clouds. It requires more knowledge of the temperature, vertical velocity within the clouds, space density and vertical extent of clouds, and the mixing process between clouds and environment. Malkus [1960] has made a preliminary attempt to construct a cumulonimbus model of the tropical cyclone.

Furthermore, in order to construct a closed dynamical model of a tropical cyclone, the functions of cumulus clouds must be specified from the other macroscopic parameters. Recent proposals of Kasahara [1964], Ooyama [1964], Charney et al. [1964], and Kuo [1964] are aiming along this line.

Developing stage. When the accumulation of heat released and carried upward by cumulus convection becomes large, the mean temperature of the upper troposphere is increased, and this leads to the formation of the warm-core structure. Because of the hydrostatic relationship, the pressure in the lower layer decreases. Once a closed low-pressure pattern is formed in the lower layer, the frictional convergence is organized within the central low-pressure area. The concentration of convergence intensifies the vertical motion and the heat release. The heating and the surface friction act to accelerate the radial circulation as is shown by (3.21). 


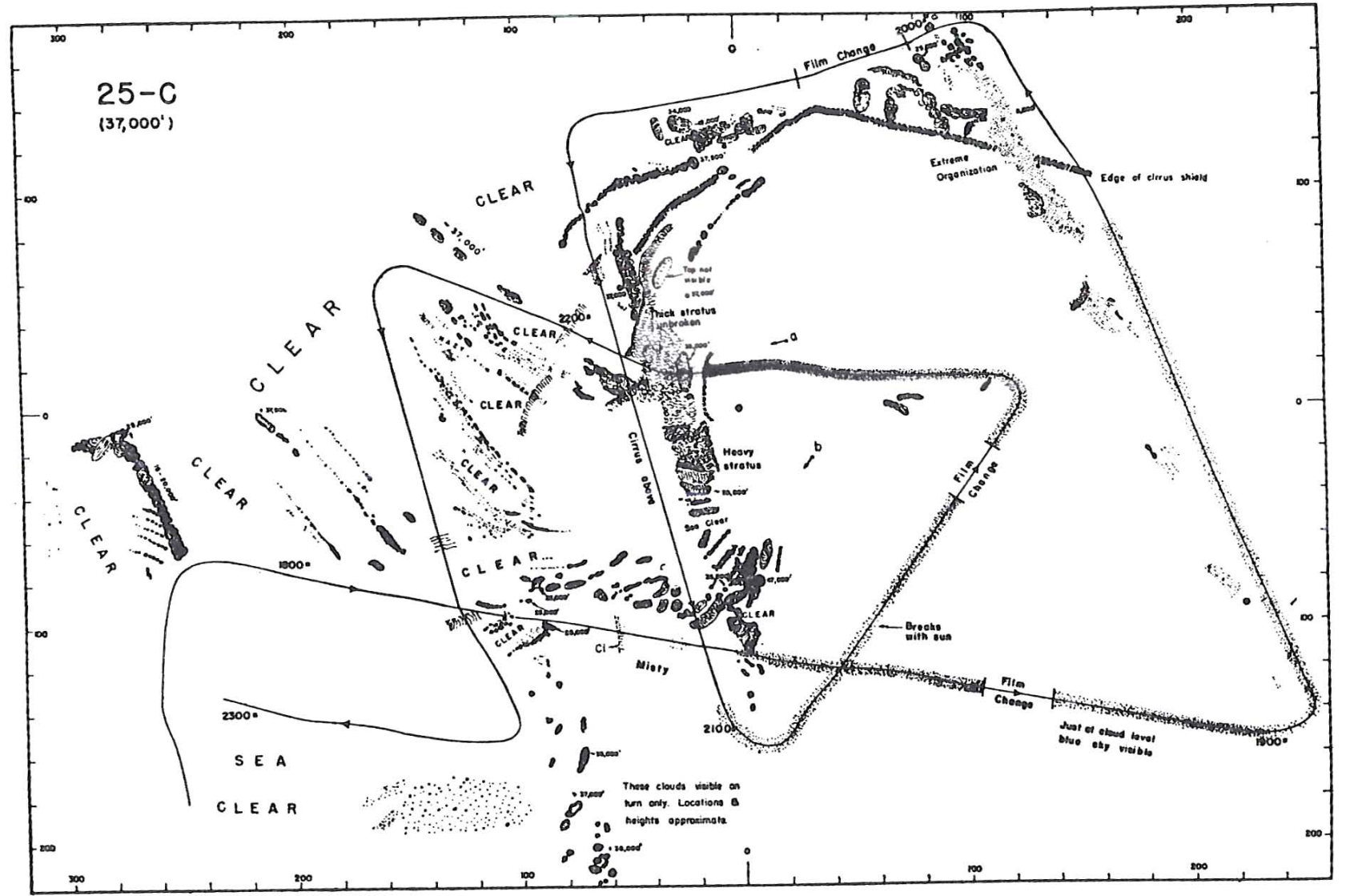

Fig. 21. Photographic cloud map for hurricane Daisy, August 25, 1958, constructed from National Hurricane research project aircraft nose camera film [after Malkus et al., 19611. 
It is not yet conclusive whether or not the inertial instability mechanism discussed in the preceding section is actually responsible for further development of a tropical cyclone. However, it is very naturally expected that the formation of a radial gradient of temperature, especially in the upper troposphere, together with the reduced static stability (see equation 4.5) and the negative relative vorticity associated with the upper anticyclone, makes the characteristic $B^{2} / S-\xi \eta$ a very small negative value or even positive. The radial and vertical circulation will be further accelerated at these conditions. It is well known that the drop of surface pressure in the tropical cyclone development is not monotonous but shows abrupt change in certain short periods [Ito, 1963].

The intensified surface inflow supplies moisture and heat from the contacting ocean surface. The cumulus convection increases, and it again produces more liberation of latent heat of condensation and its upward transport. Thus the warm core will be maintained. In this cycle of mechanisms, the circulation of a tropical cyclone may be accelerated self-excitingly until the momentum absorption in the surface boundary layer and the supply of heat reach an equilibrium state. Summarizing the postulated linkage of various mechanisms, we present the schematic diagram shown in Figure 22.

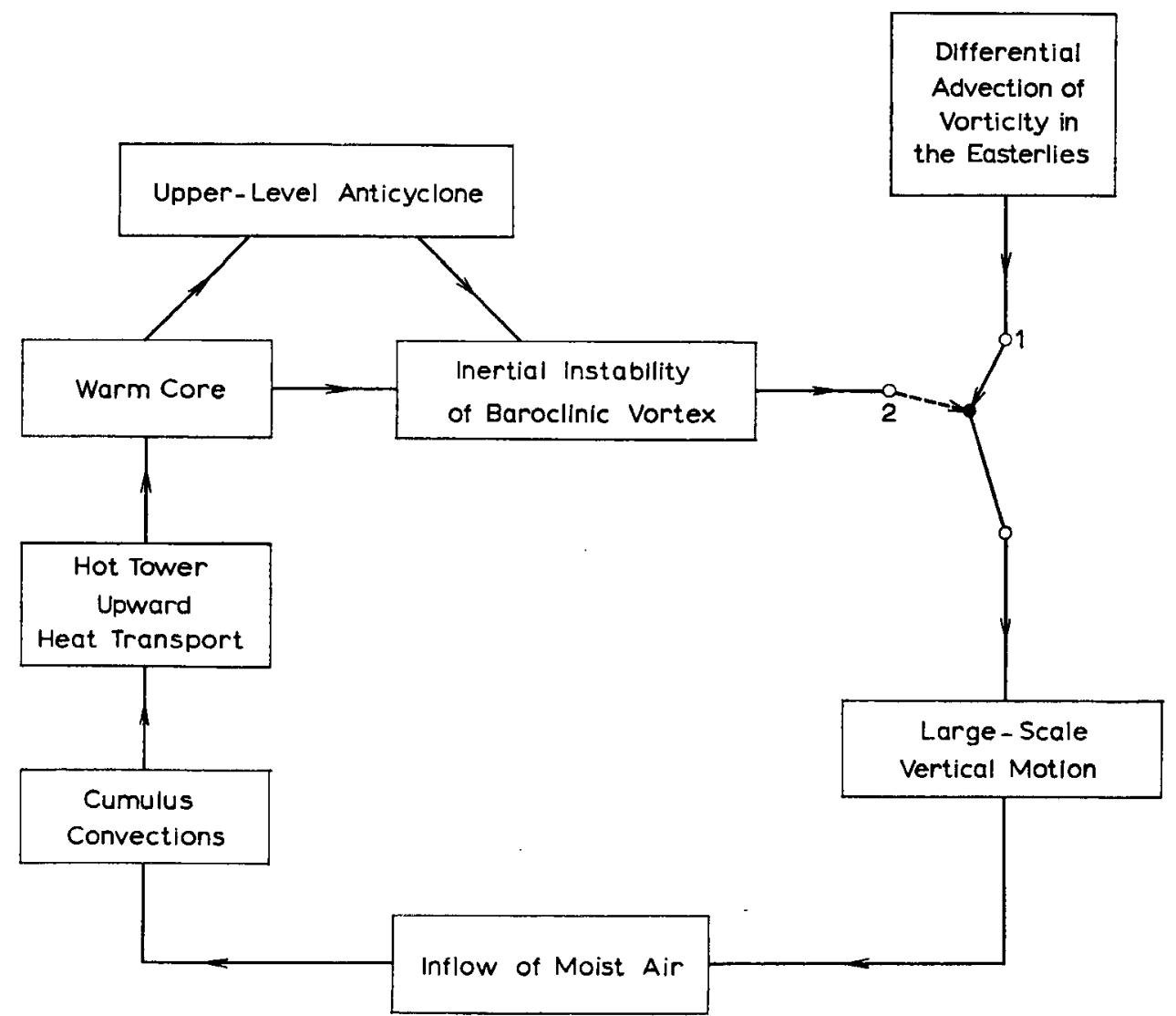

Fig. 22. Postulated linkage of various mechanisms leading to tropical cyclone formation. 


\section{OTHER RELATED STUDIES AND REMARKS}

Finally, we shall look at several other problems that are closely related to the further advance of our subject.

Mesoscale features of tropical cyclones. A basic obstacle in theoretical and numerical studies of tropical cyclone formation is that we have not acquired basic knowledge on the cumulus convection in the tropical cyclone and the mutual relationship between the clouds and the described macroscopic tropical cyclone. We do not know how much the kinetic energy is partitioned into these cloud-scale motions. We do not know in what manner the released heat is mixed with the surrounding air. For solution of this problem more observational studies on the cumulus towers in the tropical cyclone are needed. Especially, we must know the fluctuation of the vertical velocity associated with the cloud towers and its significance in the vertical transport of momentum, heat, and moisture. Gray [1964] has been attempting to measure the small-scale vertical motion and the associated vertical transport of various quantities from aircraft accelerations. Doubtless this sort of study will contribute fundamental observational knowledge for the better understanding of dynamics of the tropical cyclone.

It has been recognized that the small-scale convection embedded within the tropical cyclone is highly organized in spirally shaped bands. The bands of heavy precipitation pattern were first found by radar observation (Figure 23) [Maynard, 1945; Wexler, 1947]. There are several hypotheses on mechanisms which create the band structure of convection. Some evidence suggests that the spiral bands are related to bands or streets of clouds commonly observed over the tropical oceanic area. Aircraft photographic observation [Malkus and Ronne, 1960] and several analyses of photographs taken by meteorological satellites [Schuetz and Fritz, 1961] have

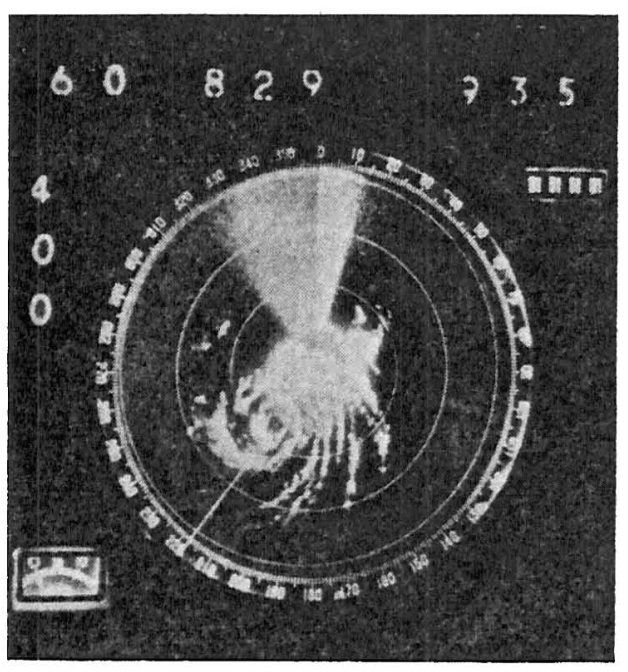

Fig. 23. Rain bands in typhoon. Della of 1960 observed from Murotomisaki weather radar station, Shikoku, Japan (Japan Meteorological Agency).

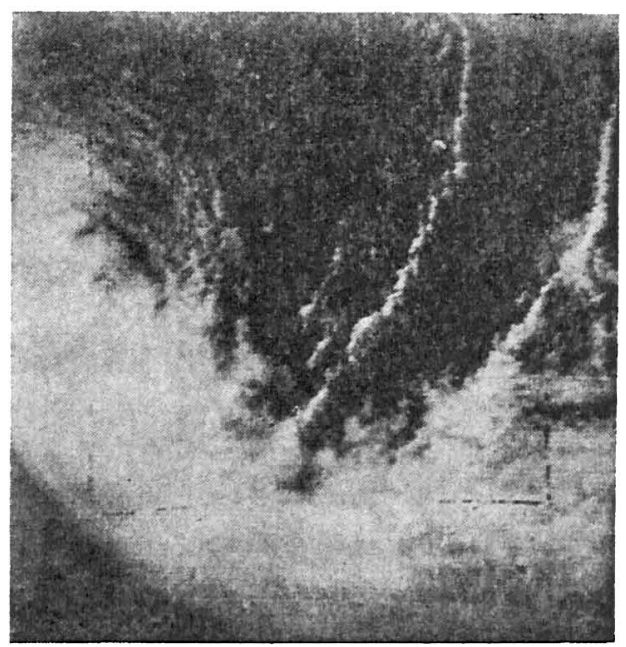

Fig. 24. Tiros picture of tropical cyclone centered north of New Zealand, April 10, 1960 [after Fritz and Wexler, 1960]. 
shown that row organization of cumulus clouds is a very common phenomenon in the trades of the tropies.

The origin of the cloud streets has been discussed by Kuettner [1959] and $K u o$ [1962b]. They hypothesized that the vertical shear in the prevailing wind leads to suppressions of the convective motion in the vertical plane parallel to the wind. Thus the convection tends to organize along the prevailing wind direction. On the other hand, Faller [1962] has proposed a mechanism unique for the spiral bands of tropical cyclones. He made a model experiment on the instability of the surface boundary layer in a rotating tank filled with water. According to his results, a kind of shearing instability occurs when the Reynolds number in the boundary layer reaches a certain value. The instability causes bandlike structure of divergence and convergence in the surface inflow.

The role and significance of the bands for the maintenance of tropical cyclones is not yet understood. Gentry [1964] has published a preliminary study describing some observations of temperature, vertical velocity, and liquid-water content across the bands.

Meteorological satellites. Recent success of meteorological satellite observations has added a powerful tool for the investigation of tropical disturbances and cloud distribution over a wide oceanic area. The satellites have made an amazing number of photographic observations and infrared radiation measurements. Some preliminary studies on the cloud distribution associated with tropical storms (Figure 24) [Fritz and Wexler, 1960; Fritz, 1962], on the equatorial convergence zone [Hubert, 1961], and on the determination of detailed topography of cloud tops in a hurricane by black-body temperature reduced from radiation measurement [Fujita and Arnold, 1964] have shown promise for new discovery and interpretation of the wide variety of problems.

Demands for more observations in the tropics. Despite all the efforts of meteorologists that are summarized in this review, the observational study of pre-existing disturbances in the tropics is still very fragmentary and qualitative. The mechanisms of origin and maintenance of easterly waves, for example, are not known. For a further advance of the study of tropical cyclone formation, quantitative analysis of pre-existing disturbances in terms of dynamical mechanism, study of the relationship between the disturbances and the general circulation of the tropical atmosphere, investigation of the equatorial shear zone, etc., are necessary. The current operational rawinsonde observation network is too poorly located in the tropics for this work. For progress of fundamental understanding of processes in the tropical atmosphere, I would like to conclude this review paper by calling the attention of researchers to this demand for more observations.

Acknowledgments. I have profited from personal discussion with many contributors to this subject matter. Sincere thanks are due Dr. H. Riehl, who carefully read the manuscript and made several valuable comments. Thanks are also extended to $\mathrm{Wm}$. Gray and $\mathrm{L}$. Pigno, who took pains to revise the original manuscript.

\section{REFERENCES CITED}

Alaka, M. A., Dynamics of upper-air outflow in incipient hurricanes, Geophysica, 6, 133-146, 1958. 
Alaka, M. A., The occurrence of anomalous winds and their significance, Monthly Weather Rev., 89, 482-494, 1961.

Alaka, M. A., On the occurrence of dynamic instability in incipient and developing hurricanes, Monthly Weather Rev., 90, 49-58, 1962.

Alaka, M. A., Instability aspects of hurricane genesis, Natl. Hurricane Res. Proj. Rept.64, 1963.

Arakawa, H., Typhoon climatology as revealed by data of the Japanese weather service, Proc. Inter-Regional Seminar on Tropical Cyclones, Tokyo, 1962, pp. 31-36, Japan Meteorological Agency, 1963.

Berkofsky, L., A numerical model for the prediction of hurricane formation, Geophys. Res. Paper 67, 1960.

Byers, H. R., General Meteorology, McGraw-Hill Book Company, New York, 645 pp., 1944.

Chandrasekhar, S., The instability of a layer of fluid heated below and subject to Coriolis forces, Proc. Roy. Soc. London, A, 217, 306-307, 1953.

Charney, J. G., A. Eliassen, and Y. Ogura, The dynamics of hurricane formation, paper presented at 3rd Tech. Conf. on Hurricanes and Trop. Meteorol., Mexico City, 1963, to be published, 1964.

Colón, J. A., and staff of the National Hurricane Research Project, On the structure of hurricane Daisy, Natl. Hurricane Res. Proj. Rept. 48, 1961.

Depperman, C. E., Outlines of Philippine Frontology, Bureau of Printing, Manila, 1936.

Dunn, C. E., Cyclogenesis in the tropical Atlantic, Bull. Am. Meteorol. Soc., 21, 215-229, 1940.

Dunn, C. E., and B. I. Miller, Atlantic Hurricanes, Louisiana State University Press, Baton Rouge, $326 \mathrm{pp}, 1960$.

Ekman, V. W., On the influence of the earth's rotation on ocean currents, Arkiv Matem. Astr. Fysik Stockholm, 2(11), 1905.

Eliassen, A., The quasi-static equations of motion with pressure as independent variable, Geofys. Publikasjoner, $17(3), 1949$.

Eliassen, A., Slow thermally or frictionally controlled meridional circulation in a circular vortex, Astrophys. Norvegica, 5(2), 1952.

Eliassen, A., On the formation of fronts in the atmosphere, in The Atmosphere and the Sea in Motion, edited by B. Bolin, Rockefeller Inst. Press, New York, 509 pp., 1959.

Estoque, M. A., Vertical and radial motions in a tropical cyclone, Tellus, 14, 394-402, 1962.

Estoque, M. A., Some numerical studies of tropical cyclones, paper presented at the 3rd Tech. Conf. on Hurricanes and Trop. Meteor., Mexico City, 1963, to be published, 1964.

Faller, A. J., An experimental analogy to and proposed explanation of hurricane spiral bands, in Proc. 2nd Tech. Conf. on Hurricanes and Trop. Meteorol., Miami Beach, 1961, Natl. Hurricane Res. Proj. Rept. 50, 307-313, 1962.

Fisher, E. L., The exchange of energy between the sea and the atmosphere in relation to hurricane behavior, Natl. Hurricane Res. Project Rept. 8, part 2, 1957.

Fjørtoft, R., Application of integral theorems in driving criteria of stability for laminar flows and for the baroclinic circular vortex, Geofys. Publikasjoner, 17 (6), 1950.

Fritz, S., Satellite pictures and the origin of hurricane Anna, Monthly Weather Rev., 90, 507513,1962 .

Fritz, S., and H. Wexler, Cloud pictures from satellite Tiros I, Monthly Weather Rev., 88, 79$87,1960$.

Fujita, T., and J. E. Arnold, The dissipating stage of hurricane Anna over the Mexican plateau as portrayed by Tiros cameras and infra-red radiometers, paper presented at 3rd Tech. Conf. on Hurricanes and Trop. Meteor., Mexico City, 1963, to be published, 1964.

Fultz, D., A survey of certain thermally and mechanically driven systems of meteorological interest, in Proc. 1st Symp. Use of Models in Geophys. Fluid Dynamics, The Johns Hopkins University, 1958, pp. 27-63, 1956.

Fultz, D., et al., Studies of thermal convection in a rotating cylinder with some implications for large-scale atmospheric motions, Meteorol. Monographs, 4(21), 104 pp., 1959.

Gabites, J. F., The origin of tropical cyclones, Proc. Inter-Regional Seminar on Tropical Cyclones, Tokyo, 1962, pp. 53-58, Japan Meteorological Agency, 1963.

Gentry, R. C., Historical survey and climatology of hurricanes and tropical storms, Proc. Inter- 
Regional Seminary on Tropical Cyclones, Tokyo, 1962, pp. 17-29, Japan Meteorological Agency, 1963.

Gentry, R. C., The role of the rainbands in hurricanes, paper presented at 3rd Tech. Conf. on Hurricanes and Trop. Meteorol., Mexico City, 1963, to be published, 1964.

Gray, W. M., A method of direct calculation of draft scale vertical motion from Research Flight Facility flight observations, paper presented at 3rd Tech. Conf. on Hurricanes and Trop. Meteorol., Mexico City, 1963, to be published, 1964.

Haque, S. M., The initiation of cyclonic circulation in a vertically unstable stagnant air mass, Quart.J. Roy. Meteorol. Soc., 78, 394-406, 1952.

Hide, R., Some experiments on thermal convection in a rotating liquid, Quart. J. Roy. Meteorol. Soc., $79,161,1953$.

Hide, R., An experimental study of thermal convection in rotating liquid, Phil. Trans. Roy. Soc. London, A, 250, 441-478, 1958.

Høiland, E., On the stability of the circular vortex, Avhandl. Norske Videnskabs-Akad. Oslo, $1(11), 1941$.

Horiguti, Y., On the typhoons of the far east, Mem. Imperial Marine Obs. Kobe, Q, 111-162, 1926.

Horiguti, Y., On the energy of a typhoon, Geophys. Mag., Tokyo, Okada Vol., 39-57, 1932.

Hubert, L. F., A case study of hurricane formation, J. Meteorol., 12, 486-492, 1955.

Hubert, I. F., A subtropical convergence line of the south Pacific, J. Geophys. Res., 66, 797$812,1961$.

Hughes, L. A., On the low-level wind structure of tropical storms, J. Meteorol., 9, 422-428, 1952.

Ito, H., Aspects of typhoon development, Proc. Inter-Regional Seminar on Tropical Cyclones, Tokyo, 1962, pp. 103-119, Japan Meteorological Agency, 1963.

Jacobs, W.C., On the energy exchange between sea and atmosphere, J. Mar. Res., 5, 37-66, 1942.

Jordan, C. L., Mean soundings for the West Indies area, J. Meteorol., 15, 91-97, 1958a.

Jordan, C. L., The thermal structure of the core of tropical cyclones, Geophysica, 6, 281-297, $1958 b$.

Jordan, C. L., and E. S. Jordan, On the mean thermal strueture of tropical cyclones, $J$. Meteorol., 11, 440-448, 1954.

Jordan, C. L., and collaborators, On the structure of hurricane Daisy on 27 August 1958, $J$. Meteorol., 17, 337-348, 1960.

Jordan, E. S., An observational study of upper wind circulation around tropical storms, $J$. Meteorol., 9, 340-346, 1952.

Kasahara, A., The structure of a tropical cyclone in the incipient stage, Geophys. Mag. Tokyo, 24, 129-154, 1952.

Kasahara, A., Supplementary notes on the formation and the schematic structure of typhoons, J. Meteorol. Soc. Japan, [2] 32, 31-52, 1954.

Kasahara, A., A numerical experiment on the development of a tropical cyclone, J. Meteorol., $18,259-282,1961 a$.

Kasahara, A., A study of stability of thermally driven and frictionally controlled symmetrical motions with application to the mechanism for development of tropical cyclones, Rept. Univ. Chicago, Dept. Geophys. Sci., 64 pp., $1961 b$.

Kasahara, A., Numerical experiments on the development of tropical cyclones, paper presented at 3rd Tech. Conf, on Hurricanes and Trop. Meteorol., Mexico City, 1963, to be published, 1964.

Kleinschmidt, E., Grundlagen einer Theorie der tropischen Zyklonen, Archiv Meteorol, Geophys., Bioklimatol., A, 4, 53-72, 1951.

Koteswaram, P., Origin of tropical storms over the Indian Ocean, Proc. Inter-Regional Seminar on Tropical Cyclones, Tokyo, 1962, pp. 69-78, Japan Meteorological Agency, 1963.

Koteswaram, P., and C. A. George, The formation and structure of tropical cyclones in the Indian Sea areas, 75th Anniversary Vol. J. Meteorol. Soc. Japan, 309-322, 1957.

Kuettner, J., The band structure of the atmosphere, Tellus, 11, 267-294, 1959. 
Kuo, H. I., Symmetrical disturbances in a thin layer of fluid subject to a horizontal temperature gradient and rotation, J. Meteorol., 11, 399-411, 1954.

Kuo, H. L., Forced and free axially symmetric convection produced by differential heating in a rotating fluid, J. Meteorol., 19, 521-527, 1956.

Kuo, H. L., Dynamics of convective vortices and eye formation, in The Atmosphere and the Sea in Motion, edited by B. Bolin, Rockefeller Inst. Press, New York, 509 pp., 1959.

Kuo, H. L., Convection in conditionally unstable atmosphere, Tellus, 18, 441-459, 1961.

Kuo, H. L., On the controlling influences of eddy diffusion on thermal convection, J. Atmospheric Sci., 19, 236-243, 1962a.

Kuo, H. L., Perturbations of plane couette flow in stratified fluid and origin of cloud-streets, Rept. Univ. Chicago, Dept. Geophys. Sci., $1962 b$.

Kuo, H. L., A balanced and an unbalanced dynamic model for the study of hurricane development, paper presented at 3rd Tech. Conf. on Hurricanes and Trop. Meteorol., Mexico City, 1963, to be published, 1964 .

Lilly, D. K., On the theory of disturbances in a conditionally unstable atmosphere, Monthly Weather Rev., 88, 1-17, 1960.

Malkus, J. S., Recent developments in studies of penetrative convection and an application to hurricane cumulonimbus towers, in Cumulus Dynamics, edited by C. E. Anderson, Pergamon Press, New York, 211 pp., 1960.

Malkus, J., and H. Riehl, On the dynamics and energy transformation in steady-state hurricanes, Tellus, 12, 1-20, 1960.

Malkus, J. S., and C. Ronne, Cloud distributions over the tropical oceans in relation to largescale flow patterns, in Physics of Precipitation, edited by H. Weickmann, American Geophysical Union, Washington, 435 pp., 1960.

Malkus, J., C. Ronne, and M. Chaffee, Cloud patterns in hurricane Daisy, 1958, Tellus, 13, 830, 1961.

Maynard, R. H., Radar and weather, J. Meteorol., 2, 214-226, 1945.

MeRae, J. N., The formation and development of tropical cyclones during the 1955-56 season in Australia, Proc. Tropical Cyclone Symposium, Brisbane, 1956, pp. 233-261, Australian Meteorological Office, 1956.

Nakagawa, Y., and P. Frenzen, A theoretical and experimental study of cellular convection in rotating fluids, Tellus, 7, 1-21, 1955.

Ooyama, K., A dynamical model for the study of tropical cyclone development, paper presented at 3rd Tech. Conf. on Hurricanes and Trop. Meteorol., Mexico City, 1963, to be published, 1964.

Otani, T., Converging line of the northeast trade wind and converging belt of the tropical air current, Geophys. Mag. Tokyo, 25, 1-122, 1953.

Palmén, E., On the formation and structure of tropical hurricanes, Geophysica, 3, 26-38, 1948.

Palmén, E., and C. L. Jordan, Note on the release of kinetic energy in tropical cyclones, Tellus, 7, 186-188, 1955.

Palmén, E., and H. Riehl, Budget of angular momentum and energy in tropical cyclones, $J$. Meteorol., 14, 150-159, 1957.

Palmer, C. E., Tropical meteorology, Quart. J. Roy. Meteorol. Soc., 78, 126-163, 1952.

Priestley, C. H. B., and W. C. Swinbank, Vertical transport of heat by turbulence in the atmosphere, Proc. Roy. Soc. London, A, 189, 543-561, 1947.

Ramage, C. S., Hurricane development, J. Meteorol., 16, 227-237, 1959.

Rayleigh, Lord, On convection currents in a horizontal layer of fluid when the higher temperature is on the under side, Phil. Mag. London, [6]32, 529-546, 1916.

Riehl, H., Waves in the easterlies and the polar front in the tropics, Dept. Meteorol., Univ. Chicago Misc. Rept. 17,79 pp., 1945.

Riehl, H., On the formation of west Atlantic hurricanes, Dept. Meteorol., Univ. Chicago Misc. Rept. 24, 1-64, 1948a.

Riehl, H., On the formation of typhoons, J. Meteorol., 5, 247-264, $1948 b$.

Riehl, H., F. Baer, and K. Veigas, Prediction of hurricane formation in the Gulf of Mexico, 
paper presented at 3rd Tech. Conf. on Hurricanes and Trop. Meteorol., Mexico City, 1963, to be published, 1964.

Riehl, H., Some relations between wind and thermal structures of steady state hurricanes, $J$. Atmospheric Sci., 20, 276-287, 1963.

Riehl, H., and R. C. Gentry, Analysis of tropical storm Frieda 1957, Natl. Hurricane Res. Proj. Rept. 17, 1958.

Riehl, H., and J. S. Malkus, On the heat balance in the equatorial trough zone, Geophysica, 6 , 503-538, 1958 .

Riehl, H., and J. S. Malkus, Some aspects of hurricane Daisy, 1958, Tellus, 13, 181-213, 1961.

Rosenthal, S. L., A simplified linear theory of equatorial easterly waves, J. Meteorol., 17, 484$488,1960$.

Rosenthal, S. L., Some numerical studies of circularly symmetric motions with application to tropical cyclones, paper presented at 3rd Tech. Conf. on Hurricanes and Trop. Meteorol., Mexico City, 1963, to be published, 1964.

Sawyer, J. S., Notes on the theory of tropical cyclones, Quart. J. Roy. Meteorol. Soc., 73, 101$126,1947$.

Schuetz, J., and S. Fritz, Cloud streets over the Caribbean Sea, Monthly Weather Rev., 89, 375-382, 1961.

Simpson, R. H., A note on the movement and structure of the Florida hurricane of October 1946, Monthly Weather Rev., 75, 53-58, 1947.

Syōno, S., Approximate solution of nonlinear differential equation of stationary wind in axial symmetric cyclone and anticyclone and its applications, Geophys. Mag. Tokyo, 20, 39-65, 1949.

Syōno, S., On the structure of atmospheric vortices, J. Meteorol., 8, 103-110, 1951.

Syono, S., On the formation of tropical cyclones, Tellus, $5,179-195,1953$.

Syōno, S., A numerical experiment of the formation of tropical cyclones, Proc. Intern. Symp. Numerical Weather Prediction, Tokyo, 1960, pp. 405-418, Meteorological Society of Japan, 1962.

Syōno, S., and I. Hirota, Numerical experiment on the development of a tropical cyclone, paper presented at Intern. Symp. Dynamics of Large-Scale Processes, Boulder, 1963.

Syōno, S., Y. Ogura, K. Gambo, and A. Kasahara, On the negative vorticity in a typhoon, $J$. Meteorol. Soc. Japan, [2]12, 1-19, 1951.

Taylor, G. I., Experiments with rotating fluids, Proc. Roy. Soc. London, A, 100, 114-121, 1921.

Terada, K., Development of typhoon from the viewpoint of energy supply from ocean, in Proc. UNESCO Symp. Typhoons, Tokyo, 1954, pp. 199-206, Japanese National Commission for UNESCO, 1955.

Uwai, K., Studies on the equatorial front in the Micronesian Islands and its relation to typhoons and squalls (in Japanese), Secret Meteorol. Rept., Central Meteorol. Observatory, Tokyo, 1, 237-273, 1943.

Wexler, H., Structure of hurricanes as determined by radar, Ann N. Y. Acad. Sci., 48, 821-845, 1947.

Yanai, M., A detailed analysis of typhoon formation, J. Meteorol. Soc. Japan, [2]s9, 187-214, $1961 a$.

Yanai, M., Dynamical aspects of typhoon formation, J. Meteorol. Soc. Japan, [2]39, 282-309, $1961 b$.

Yanai, M., A comment on the creation of warm core in incipient tropical cyclone, Typhoon Res. Lab., Meteorol. Res. Inst., Tokyo, Tech. Note 1, 1963.

Yanai, M., A preliminary survey of large-scale disturbances over the tropical Pacific, paper presented at 3rd Tech. Conf. on Hurricanes and Trop. Meteorol., Mexico City, 1963, to be published, 1964 .

\section{GENERAL REFERENCES}

Chandrasekhar, S., Hydrodynamic and Hydromagnetic Stability, Oxford University Press, London, 652 pp., 1961. 
Dunn, G. E., and B. I. Miller, Atlantic Hurricanes, Louisiana State University Press, Baton Rouge, $326 \mathrm{pp} ., 1960$.

Eliassen, A., and E. Kleinschmidt, Jr., Dynamic meteorology, Handbuch der Physik, 48, Springer-Verlag, Berlin, 1045 pp., 1957.

Fritz, S., Research with satellite cloud pictures, Astronautics Aerospace Eng., 1(3), 70-75, 1963.

Gentry, R. C., Origins of tropical cyclones, in Proc. Inter-Regional Seminar on Trop. Cyclones, Tokyo, 1962, pp. 59-68, Japan Meteorological Agency, $1963 a$.

Gentry, R. C., Structure of tropical storms, in Proc. Inter-Regional Seminar on Trop. Cyclones, Tokyo, 1962, pp. 133-158, Japan Meteorological Agency, 19636.

Masuda, Y., and A. Kasahara, Theory of typhoons (in Japanese), in Lectures on Meteorology, Vol. 11, Chijin Shokan, Tokyo, 138 pp., 1956.

Palmén, E., Formation and development of tropical cyclones, Proc. Trop. Cyclone Symp. Brisbane, 1956, pp. 213-231, 1956.

Palmer, C. E., Tropical meteorology, Quart. J. Roy. Meteorol. Soc., 78, 126-164, 1952.

Riehl, H., A model of hurricane formation, J. Appl. Phys., 21, 917-925, 1950.

Riehl, H., Aerology of tropical storms, in Compendium of Meteorology, edited by T. F. Malone, American Meteorological Society, 1334 pp., 1951.

Riehl, H., Tropical Meteorology, MeGraw-Hill Book Company, New York, 392 pp., 1954.

Richl, H., On the origin and possible modification of hurricanes, Science, 141 (3585), 1001-1010, 1963.

Saltzman, B., editor, Selected Papers on Theory of Thermal Convection, Dover Publications, 461 pp., 1962.

Syōno, S., Formation of tropical cyclones, in Proc. Inter-Regional Seminar on Tropical Cyclones, Tokyo, 1962, pp. 79-93, Japan Meteorological Agency, 1963.

Yanai, M., On the formation of typhoons, 1, 2, (in Japanese), Kagaku (Science), 82, no. 9 and no. 11, Iwanami Shoten, Tokyo, 1962.

(Manuscript received October 15, 1963.) 